Check for updates

Cite this: RSC Adv., 2019, 9, 12255

\title{
Protecting group-directed annulations of tetra- substituted oxindole olefins and sulfur ylides: regio- and chemoselective synthesis of cyclopropane- and dihydrofuran-fused spirooxindoles $\dagger$
}

\author{
Jing-Wen Kang,,$^{a}$ Xiang Li,,$^{a}$ Fei-Yu Chen, ${ }^{a}$ Yuan Luo, ${ }^{a}$ Shu-Cang Zhang, ${ }^{a}$ Bin Kang, ${ }^{a}$ \\ Cheng Peng, (D) ${ }^{a}$ X Tian ${ }^{* b}$ and Bo Han (D) *a
}

Protecting group-controlled annulations of tetra-substituted oxindole olefins and sulfur ylides have been achieved for the synthesis of multifunctional cyclopropane- and dihydrofuran-fused spirooxindoles. Under precise annulation regulation, a variety of cyclopropane- and dihydrofuran-fused spirooxindoles containing vicinal quaternary carbon centers were produced in up to $90 \%$ yield with up to $20: 1 \mathrm{dr}$. This reaction demonstrates high regio-, chemo- and diastereoselectivity, broad functional group tolerance and gram-scale capacity.

Received 21st March 2019

Accepted 10th April 2019

DOI: 10.1039/c9ra02192b

rsc.li/rsc-advances

We used sulfur ylide chemistry to construct structurally complex three- and five-membered spirooxindoles containing vicinal quaternary carbon centers. As a versatile and efficient synthetic tool, sulfur ylide can facilitate formation of cyclic fragments. ${ }^{6}$ Mono- or di-substituted double bond substrates tend to generate small ring compounds via the [2 $+1]$ pathway, such as cyclopropane, epoxy and aziridine (Scheme 1a, left column). ${ }^{7}$ Tri- or tetra-substituted double bond substrates can generate five-membered heterocyclic compounds via the $[4+1]$ pathway, such as dihydrofuran, dihydropyrrole and indolin (Scheme 1a, right column). ${ }^{8}$ Trior tetra-substituted double bond substrates can also groups; such carbon on the ring with multiple function are important for biological activity (Fig. 1b). ${ }^{4}$ These centers are difficult to build and modify with functional groups because steric hindrance and substrate reactivity strongly affect formation of continuous quaternary

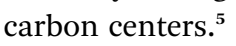

${ }^{a}$ State Key Laboratory Breeding Base of Systematic Research Development and Utilization of Chinese Medicine Resources School of Pharmacy, Chengdu University of Traditional Chinese Medicine, Chengdu 611137, P. R. China. E-mail: hanbo@ cdutm.edu.cn

${ }^{b}$ Key Laboratory of Molecular Target \& Clinical Pharmacology and the State Key Laboratory of Respiratory Disease, School of Pharmaceutical Sciences \& the Fifth Affiliated Hospital, Guangzhou Medical University, Guangzhou 511436, P. R. China. E-mail: xtian@gzhmu.edu.cn

$\uparrow$ Electronic supplementary information (ESI) available: Detailed experimental procedures and full spectroscopic data of all the compounds. CCDC 1895883 and 1895884. For ESI and crystallographic data in CIF or other electronic format see DOI: 10.1039/c9ra02192b

$\ddagger$ These authors contributed equally to this work.

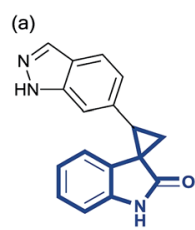

PLK 4 inhibitor

(b)

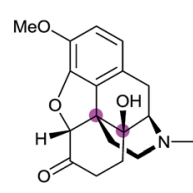

oxycodone

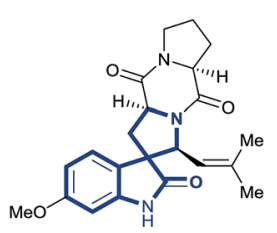

spirotryprostain A

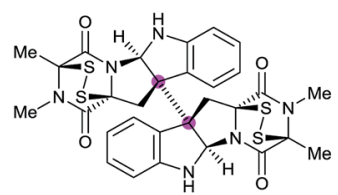

$11,11^{\prime}$ - dideoxyverticillin $\mathrm{A}$

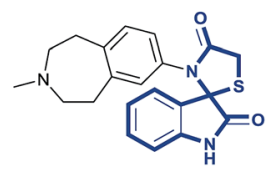

5-HT6 antagonist

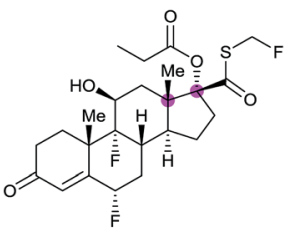

fluticasone

Fig. 1 (a) Natural products and drugs contain a three- and fivemembered spirooxindole scaffold and (b) drug molecules contain vicinal quaternary carbon centers. 
(a) Annulations of sulfur ylides

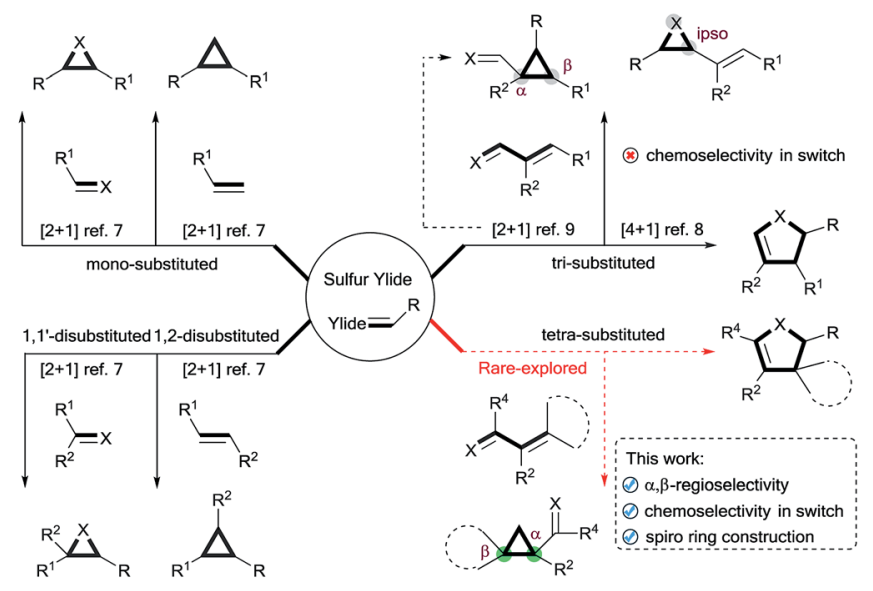

(b) Constructions of spirooxindoles
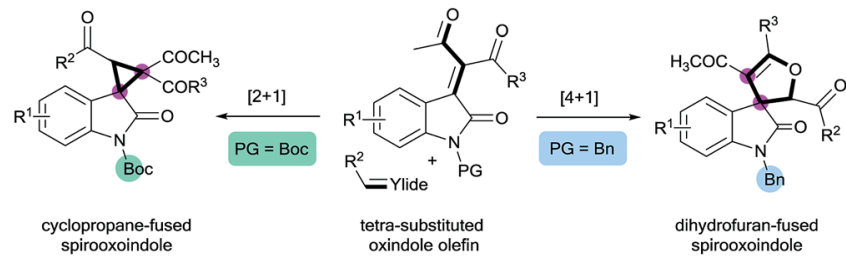

cyclopropane-fused spirooxoindole

(1) vicinal quaternary carbon centers

(1) chemo- and regioselectivity regulation $\bigcirc$ mild condition

Scheme 1 (a) Annulations between various substrates and sulfur ylides and (b) construction of cyclopropane- and dihydrofuran-fused spirooxindoles containing vicinal quaternary stereocenters. undergo the $[2+1]$ pathway to construct a three-membered ring, albeit with different regioselectivity: the trisubstituted substrate participates in the $[2+1]$ pathway with ipso or $\alpha, \beta$ selectivity; ${ }^{9}$ the tetra-substituted substrate, with $\alpha, \beta$-selectivity (Scheme 1a, right column, lower panel). ${ }^{\mathbf{1 0}}$ The tetra-substituted substrate can directly establish quaternary carbon centers in one step, as well as control the chemoselectivity between $[2+1]$ and $[4+1]$ pathways. In addition, tetra-substituted double bond substrates can be used to construct the spiro ring. Nevertheless, their low reactivity and steric hindrance have limited their synthetic use.

Despite the advances in using sulfur ylide as a synthetic tool, few reports have focused on using it to form rings of desired size in a chemo- and regioselective manner. ${ }^{11}$ This reflects, at least in part, uncertainty in how to control the cycloaddition pathway in a one-step reaction. Achieving chemo- and regioselective ring formation would enrich molecular libraries and accelerate new drug development. Therefore, we chose a tetra-substituted oxindole olefin as substrate to synthesize multi-substituted, three- and five-membered spirooxindoles containing vicinal quaternary carbon centers via sulfur ylide, as part of our continuing interest in synthesis of drug-like scaffolds. ${ }^{\mathbf{1 2}}$ Installing different protecting groups on the tetra-substituted substrate makes the synthesis chemoselective (Scheme 1b). This reaction allows the production of desirable cyclopropaneor dihydrofuran-fused spiro-oxoindoles in high yield with high chemo-, regio- and diastereoselectivities.

Table 1 Optimization of the reaction conditions ${ }^{a}$

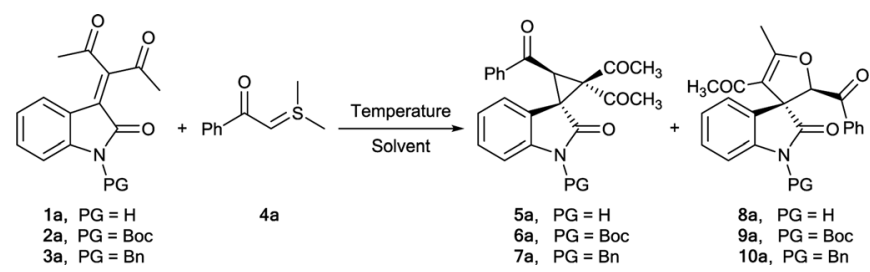

\begin{tabular}{llllll}
\hline Entry & PG & Solvent & Temperature $\left({ }^{\circ} \mathrm{C}\right)$ & Yield $^{b}(\%)(\mathbf{6 a} / \mathbf{9 a})$ & Yield $(\%)(\mathbf{7 a} / \mathbf{1 0 a})$ \\
\hline 1 & H & MeCN & 25 & $45(\mathbf{5 a})$ & - \\
2 & Boc & MeCN & 25 & $48 / 21$ & - \\
3 & Boc & THF & 25 & Trace & - \\
4 & Boc & Tol & 25 & $68 / 17$ & - \\
5 & Boc & DCM & 25 & $82 / 9$ & - \\
6 & Boc & DCM & 50 & - & $19 / 48$ \\
7 & Bn & MeCN & 25 & - & Trace \\
8 & Bn & THF & 25 & - & $14 / 58$ \\
9 & Bn & Tol & 25 & - & $11 / 67$ \\
10 & Bn & DCM & 25 & - & $15 / 74$ \\
11 & Bn & DCM & 50 & 70 & $9 / 61$
\end{tabular}

${ }^{a}$ All reactions were carried out with $0.15 \mathrm{mmol}$ of the substrate $1 \mathrm{a} / \mathbf{2 a} / \mathbf{3 a}, 0.165 \mathrm{mmol}$ of $4 \mathrm{a}$ in $2.0 \mathrm{~mL}$ of solvent, unless otherwise stated; d.r. was determined to be $>8: 1$ by ${ }^{1} \mathrm{H}$-NMR analysis of the crude reaction mixture. ${ }^{b}$ Yields of the isolated products $6 \mathbf{a}$ and 9 a. ${ }^{c}$ Yields of the isolated products $7 \mathbf{a}$ and $10 a$. 
Table 2 Synthesis of cyclopropane-fused spirooxindole ${ }^{a}$

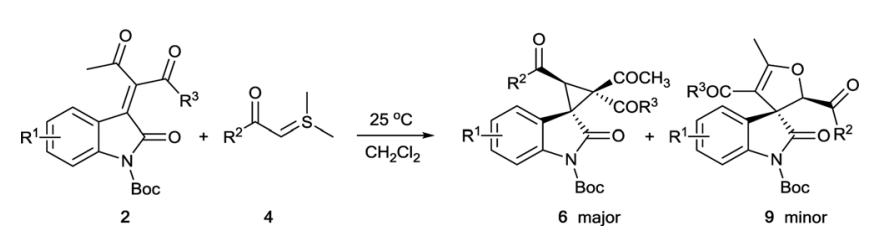

\begin{tabular}{llllll}
\hline Entry & $\mathrm{R}^{1}$ & $\mathrm{R}^{2}$ & $\mathrm{R}^{3}$ & Yield $^{b}(\%)(\mathbf{6} / 9)$ & d.r. \\
\hline 1 & $\mathrm{H}$ & $\mathrm{Ph}$ & $\mathrm{CH}_{3}$ & $82 / 9$ & $20: 1(\mathbf{6 a})$ \\
2 & $\mathrm{H}$ & $2-\mathrm{FC}_{6} \mathrm{H}_{4}$ & $\mathrm{CH}_{3}$ & $74 / 10$ & $18: 1(\mathbf{6 b})$ \\
3 & $\mathrm{H}$ & $4-\mathrm{FC}_{6} \mathrm{H}_{4}$ & $\mathrm{CH}_{3}$ & $70 / 8$ & $20: 1(\mathbf{6 c})$ \\
4 & $\mathrm{H}$ & $3,4-\mathrm{Cl}_{2} \mathrm{C}_{6} \mathrm{H}_{3}$ & $\mathrm{CH}_{3}$ & $63 / 10$ & $20: 1(\mathbf{6 d})$ \\
5 & $\mathrm{H}$ & $4-\mathrm{BrC}_{6} \mathrm{H}_{4}$ & $\mathrm{CH}_{3}$ & $75 / 12$ & $18: 1(\mathbf{6 e})$ \\
6 & $\mathrm{H}$ & $2-\mathrm{CH}_{3} \mathrm{C}_{6} \mathrm{H}_{4}$ & $\mathrm{CH}_{3}$ & $80 / 9$ & $20: 1(\mathbf{6 f})$ \\
7 & $\mathrm{H}$ & $4-\mathrm{OCH}_{3} \mathrm{C}_{6} \mathrm{H}_{4}$ & $\mathrm{CH}_{3}$ & $81 / 7$ & $20: 1(\mathbf{6 g})$ \\
8 & $\mathrm{H}$ & $\mathrm{Thienyl}$ & $\mathrm{CH}_{3}$ & $68 /$ Trace & $20: 1(\mathbf{6 h})$ \\
9 & $\mathrm{H}$ & $\mathrm{Naphthyl}$ & $\mathrm{CH}_{3}$ & $65 /$ Trace & $10: 1(\mathbf{6 i})$ \\
10 & $\mathrm{H}$ & $\mathrm{OEt}$ & $\mathrm{CH}_{3}$ & $79 / 8$ & $20: 1(\mathbf{6 j})$ \\
11 & $5-\mathrm{F}$ & $\mathrm{Ph}$ & $\mathrm{CH}_{3}$ & $68 / 13$ & $13: 1(\mathbf{6 k})$ \\
12 & $7-\mathrm{F}$ & $\mathrm{Ph}$ & $\mathrm{CH}_{3}$ & $70 / 10$ & $20: 1(\mathbf{6 1})$ \\
13 & $5-\mathrm{Cl}$ & $\mathrm{Ph}$ & $\mathrm{CH}_{3}$ & $72 / 10$ & $20: 1(\mathbf{6 m})$ \\
14 & $6-\mathrm{Cl}$ & $\mathrm{Ph}$ & $\mathrm{CH}_{3}$ & $79 / 12$ & $20: 1(\mathbf{6 n})$ \\
15 & $5-\mathrm{Br}$ & $\mathrm{Ph}$ & $\mathrm{CH}_{3}$ & $80 / 5$ & $15: 1(\mathbf{6 o})$ \\
16 & $6-\mathrm{Br}$ & $\mathrm{Ph}$ & $\mathrm{CH}_{3}$ & $81 / 8$ & $9: 1(\mathbf{6 p})$ \\
17 & $5-\mathrm{CH} \mathrm{H}_{3}$ & $\mathrm{Ph}$ & $\mathrm{CH}_{3}$ & $85 /$ Trace & $15: 1(\mathbf{6 q})$ \\
$18^{d}$ & $\mathrm{H}$ & $\mathrm{Ph}$ & $\mathrm{OEt}^{e}$ & $90 /$ Trace & $18: 1(\mathbf{6 r})$ \\
$19^{e}$ & $\mathrm{H}$ & $\mathrm{Ph}$ & $\mathrm{CH}_{3}$ & $80 / 10$ & $18: 1(\mathbf{6 a})$
\end{tabular}

${ }^{a}$ Unless otherwise noted, all reactions were performed with $2(0.15 \mathrm{mmol})$, $4(0.165 \mathrm{mmol})$ in $2 \mathrm{~mL} \mathrm{DCM}$ at $25^{\circ} \mathrm{C}$ for $2 \mathrm{~h}^{b}{ }^{b}$ Isolated yields of the major compound 6 and minor 9. ${ }^{c}$ The diastereoselective ratio of compounds 6 were calculated based on ${ }^{1} \mathrm{H}-\mathrm{NMR}$ analysis of the crude reaction mixture. ${ }^{d}$ The relative configuration of $\mathbf{6 r}$ was determined by X-ray crystallographic analysis (Fig. 2), and the relative configurations of other products 6 were tentatively assigned by analogy. ${ }^{e}$ A gram scale reaction of $2 \mathrm{a}(3.04 \mathrm{mmol})$ and $4 \mathrm{a}(3.34 \mathrm{mmol})$ in DCM at $25{ }^{\circ} \mathrm{C}$ was carried out.

\section{Results and discussion}

First, we took a tetra-substituted oxindole olefin without protecting group 1a and sulfur ylide $\mathbf{4 a}$ to conduct a model reaction in MeCN at ambient temperature. Two products with different ring sizes formed in one step with similar yields $(5 \mathbf{a}: \mathbf{8 a}=$ $1.1: 1$, Table 1 , entry 1 ). Therefore, we set out to control the chemo- and regioselectivity of the reaction and thereby influence their relative abundance. First of all, a broad type of protecting groups were tested, including methyl (-Me), allyl, acetyl (-Ac), benzyl (-Bn) and tert-butyloxycarbonyl (-Boc) groups (see Table S1 in ESI†). To our delight, changing the $\mathrm{N}-\mathrm{H}$ (1a) into $\mathrm{N}$ Boc (2a) generated 6a as a major product and 9a as a minor one (Table 1, entry 2). Using $N$-Bn (3a) produced 10a as a major product and $7 \mathbf{a}$ as a minor one (entry 7 ). Since yields were relatively low in $\mathrm{MeCN}$, these initial results encouraged us to screen solvents and temperature (entries 3-6 and 8-12). The best conditions for generation of $\mathbf{6 a}$ as the major product was in DCM at ambient temperature for about $2 \mathrm{~h}$ (entry 5). The best conditions for generation of $\mathbf{1 0 a}$ as the major product was in DCM at $50{ }^{\circ} \mathrm{C}$ for nearly $4 \mathrm{~h}$ (entry 11 ). In other words, when the reaction with $4 \mathbf{a}$ was conducted in DCM at ambient temperature generating cyclopropane-fused spirooxindole $6 \mathbf{a}$ in $82 \%$ yield.
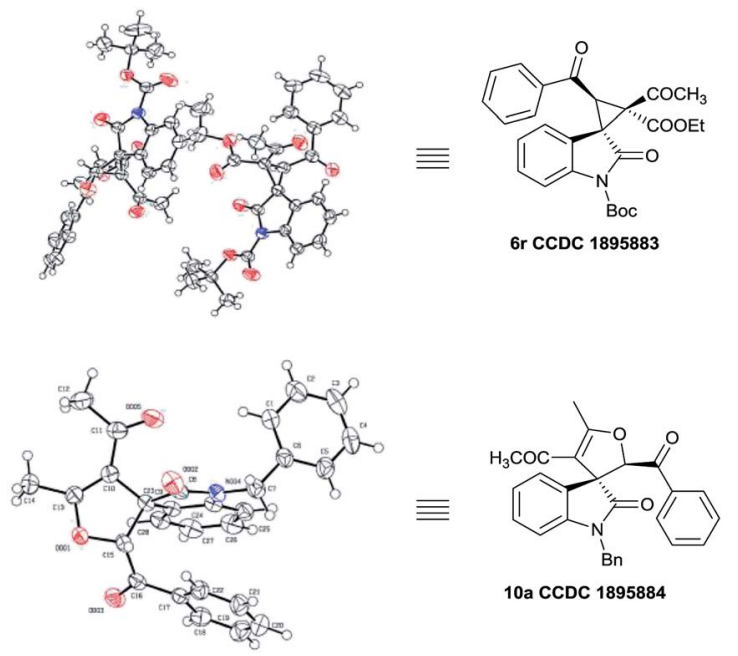

Fig. 2 Determination of relative configuration of products $6 r$ and $10 a$ by single-crystal X-ray analysis.

When the reaction was conducted with $4 \mathrm{a}$ in DCM at $50{ }^{\circ} \mathrm{C}$, 3a preferred the $[4+1]$ pathway, giving the dihydrofuran-fused spirooxindole 10a in $74 \%$ yield.

Table 3 Synthesis of dihydrofuran-fused spirooxindole ${ }^{a}$

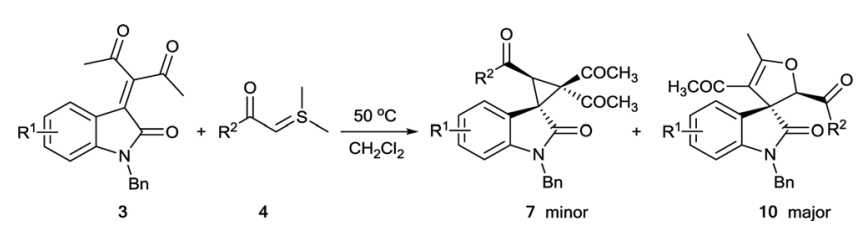

\begin{tabular}{lllll}
\hline Entry & $\mathrm{R}^{1}$ & $\mathrm{R}^{2}$ & Yield $^{b}(\%)(7 / 10)$ & d.r. $^{c}$ \\
\hline $1^{d}$ & $\mathrm{H}$ & $\mathrm{Ph}$ & $15 / 74$ & $10: 1(\mathbf{1 0 a})$ \\
2 & $\mathrm{H}$ & $2-\mathrm{FC}_{6} \mathrm{H}_{4}$ & $13 / 66$ & $16: 1(\mathbf{1 0 b})$ \\
3 & $\mathrm{H}$ & $4-\mathrm{FC}_{6} \mathrm{H}_{4}$ & $11 / 68$ & $16: 1(\mathbf{1 0 c})$ \\
4 & $\mathrm{H}$ & $3,4-\mathrm{Cl}_{2} \mathrm{C}_{6} \mathrm{H}_{3}$ & $12 / 71$ & $8: 1(\mathbf{1 0 d})$ \\
5 & $\mathrm{H}$ & $4-\mathrm{BrC}_{6} \mathrm{H}_{4}$ & $10 / 75$ & $10: 1(\mathbf{1 0 e})$ \\
6 & $\mathrm{H}$ & $2-\mathrm{CH}_{3} \mathrm{C}_{6} \mathrm{H}_{4}$ & $8 / 78$ & $20: 1(\mathbf{1 0 f})$ \\
7 & $\mathrm{H}$ & $4-\mathrm{OCH}_{3} \mathrm{C}_{6} \mathrm{H}_{4}$ & Trace/81 & $18: 1(\mathbf{1 0 g})$ \\
8 & $\mathrm{H}$ & Thienyl & $15 / 60$ & $5: 1(\mathbf{1 0 h})$ \\
9 & $\mathrm{H}$ & $\mathrm{Naphthyl}$ & $15 / 62$ & $5: 1(\mathbf{1 0 i})$ \\
10 & $\mathrm{H}$ & $\mathrm{CH}$ & $14 / 74$ & $6: 1(\mathbf{1 0 j})$ \\
11 & $\mathrm{H}$ & $\mathrm{OEt}$ & $13 / 76$ & $5: 1(\mathbf{1 0 k})$ \\
12 & $5-\mathrm{F}$ & $\mathrm{Ph}$ & $8 / 70$ & $10: 1(\mathbf{1 0 l})$ \\
13 & $7-\mathrm{F}$ & $\mathrm{Ph}$ & Trace $/ 56$ & $16: 1(\mathbf{1 0 m})$ \\
14 & $5-\mathrm{Cl}$ & $\mathrm{Ph}$ & $14 / 66$ & $5: 1(\mathbf{1 0 n})$ \\
15 & $6-\mathrm{Cl}$ & $\mathrm{Ph}$ & $12 / 67$ & $15: 1(\mathbf{1 0 0})$ \\
16 & $5-\mathrm{Br}$ & $\mathrm{Ph}$ & $10 / 70$ & $13: 1(\mathbf{1 0 p})$ \\
17 & $6-\mathrm{Br}$ & $\mathrm{Ph}$ & $10 / 69$ & $10: 1(\mathbf{1 0 q})$ \\
18 & $5-\mathrm{CH}$ & $\mathrm{Ph}$ & $8 / 80$ & $18: 1(\mathbf{1 0 r})$ \\
$19^{e}$ & $\mathrm{H}$ & $\mathrm{Ph}$ & $12 / 80$ & $9: 1(\mathbf{1 0 a})$
\end{tabular}

${ }^{a}$ Unless otherwise noted, all reactions were performed with $3(0.15 \mathrm{mmol})$, $4(0.165 \mathrm{mmol})$ in $2 \mathrm{~mL} \mathrm{DCM}$ at $50{ }^{\circ} \mathrm{C}$ for $4 \mathrm{~h} .{ }^{b}$ Isolated yields of the compound 7 and $10 .{ }^{c}$ The diastereoselective ratio of compounds $\mathbf{1 0}$ were determined by ${ }^{1} \mathrm{H}$-NMR analysis of the crude reaction mixture. ${ }^{d}$ The relative configuration of $10 \mathrm{a}$ was determined by X-ray crystallographic analysis (Fig. 2), and the relative configurations of other products 10 were tentatively assigned by analogy. ${ }^{e} \mathrm{~A}$ gram scale reaction of $3 \mathrm{a}(3.13 \mathrm{mmol})$ and $4 \mathrm{a}(3.44 \mathrm{mmol})$ in DCM at $50{ }^{\circ} \mathrm{C}$ was carried out. 
Having established optimal reaction conditions (Table 1, entry 5), we examined the scope of the reaction by varying the $\mathrm{R}^{1}$ and $\mathrm{R}^{3}$ groups of 2 , as well as the $\mathrm{R}^{2}$ moiety of 4 . Various sulfur ylides with a wide range of ortho-, meta-, and para-substituents with different electronic properties were explored (Table 2, entries 2-10). The compounds were well tolerated and afforded 6b-6g in modest to high yields, and they all demonstrated good to excellent chemo- and diastereoselectivity. This indicated that the Boc protecting group favors formation of the cyclopropanefused spirooxindole 6. Moreover, sulfur ylide reacted well with linear and heterocycle substrates (entries 8-10). Different substituents on the aryl ring of 2 gave compound 6 in reasonable yields (entries 11-17); yield was better with an electron-donating substituent than with an electron-withdrawing one (entry 17). Replacing $\mathrm{R}^{3}$ with an OEt group led exclusively to $\mathbf{6 r}$ (entry 18). The relative configuration of $\mathbf{6 r}$ was determined by X-ray crystallographic analysis (Fig. 2), and the relative configurations of other products 6 were tentatively assigned by analogy. ${ }^{13}$ Finally, to evaluate the synthetic potential of this methodology, a gram scale reaction of 6a was carried out. $2 \mathrm{a}(3.04 \mathrm{mmol})$ and $4 \mathrm{a}(3.34 \mathrm{mmol})$ went smoothly in DCM at ambient temperature, affording the desired product $6 \mathbf{6}$ in $80 \%$ yield and $18: 1 \mathrm{dr}$ (entry 19).

Next we studied the reaction's generality and limitations when generating product 10. Under the optimal conditions (Table 1, entry 11), numerous sulfur ylides reacted smoothly with compounds 3 to deliver good to high yields (Table 3, entries 2-11), although a 2-F group led to slight loss of diastereoselectivity (entry 2). Sulfur ylides with linear or heterocycle substitutions performed well (entries 8-11). In all cases, the reaction showed chemo- and diastereoselectivity in forming dihydrofuran-fused spirooxindole 10. These results support Bn as a protecting group that favors formation of compound $\mathbf{1 0 .}$ Diverse substituents on the aryl ring of 3 led to smooth reaction with 4 , giving the corresponding compounds 10l-10r in 56-80\%

Table 4 Attempt to asymmetric catalytic synthesis of chiral products ${ }^{a}$
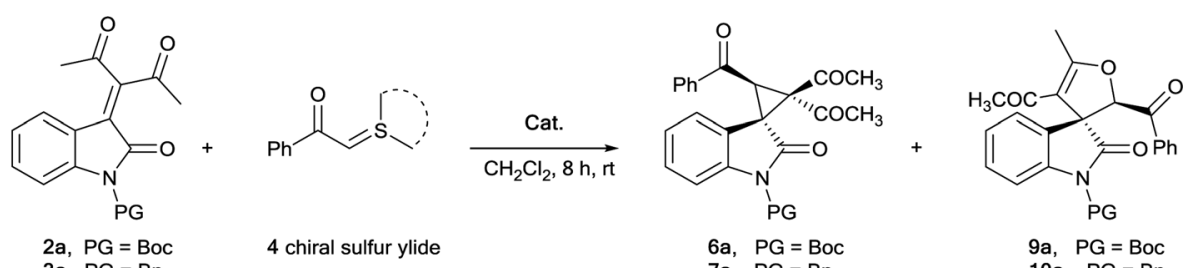

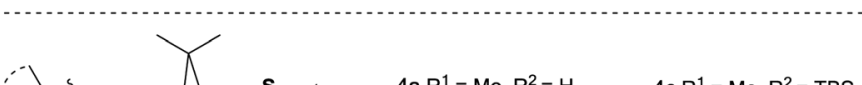<smiles>[R]SC1C2CCC(C)(C1[R20])C2(C)C#C[As]1CCCC1</smiles>

$\begin{aligned} 4 a R^{1} & =M e, R^{2}=H \\ 4 b R^{1} & =M e, R^{2}=M e\end{aligned}$

$4 c R^{1}=M e, R^{2}=T B S$
$4 d R^{1}=B n, R^{2}=M e$

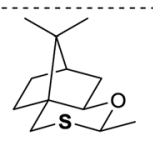

a, $P G=B n$

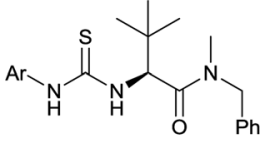

Cat. 1

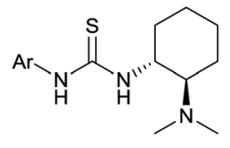

Cat. 2

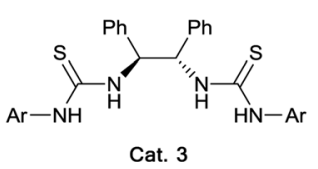

$4 \mathrm{e}$
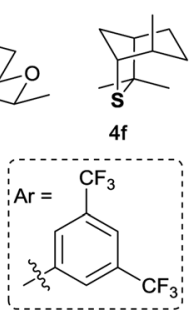

\begin{tabular}{|c|c|c|c|c|c|c|c|}
\hline Entry & PG & Sulfur ylide & Cat. & Yield $^{b}(\%)(\mathbf{6 a} / 9 \mathbf{a})$ & e.e. ${ }^{d}(\%)$ & Yield $^{c}(\%) .(7 \mathbf{a} / \mathbf{1 0 a})$ & e.e. ${ }^{e}(\%$ \\
\hline 1 & Boc & Chiral 4a & - & $61 / 13$ & 18 & - & - \\
\hline 2 & Boc & Chiral $\mathbf{4 b}$ & - & - & - & - & - \\
\hline 3 & Boc & Chiral 4c & - & - & - & - & - \\
\hline 4 & Boc & Chiral 4d & - & Trace & - & - & - \\
\hline 5 & Boc & Chiral 4e & - & $35 / 16$ & - & - & - \\
\hline 6 & Boc & Chiral $4 f$ & - & $56 / 12$ & 10 & - & - \\
\hline 7 & Boc & Chiral $4 a$ & C1 & $78 / 10$ & 25 & - & - \\
\hline 8 & Boc & Chiral $4 a$ & $\mathrm{C} 2$ & $68 / 17$ & 20 & - & - \\
\hline 9 & Boc & Chiral 4a & C3 & $77 / 16$ & 39 & - & - \\
\hline 10 & $\mathrm{Bn}$ & Chiral $4 a$ & - & - & - & $16 / 53$ & 19 \\
\hline 11 & $\mathrm{Bn}$ & Chiral $\mathbf{4 b}$ & - & - & - & - & - \\
\hline 12 & $\mathrm{Bn}$ & Chiral 4c & - & - & - & - & - \\
\hline 13 & $\mathrm{Bn}$ & Chiral 4d & - & - & - & Trace & - \\
\hline 14 & $\mathrm{Bn}$ & Chiral 4e & - & - & - & $13 / 41$ & - \\
\hline 15 & $\mathrm{Bn}$ & Chiral $4 f$ & - & - & - & $17 / 59$ & 8 \\
\hline 16 & $\mathrm{Bn}$ & Chiral $4 f$ & C1 & - & - & $12 / 76$ & 17 \\
\hline 17 & $\mathrm{Bn}$ & Chiral $4 f$ & $\mathrm{C} 2$ & - & - & $18 / 65$ & 13 \\
\hline 18 & $\mathrm{Bn}$ & Chiral $4 f$ & C3 & - & - & $13 / 72$ & 32 \\
\hline
\end{tabular}

${ }^{a}$ Reactions were performed with $2 \mathrm{a}$ or $3 \mathrm{a}(0.1 \mathrm{mmol}), \mathbf{4}(0.1 \mathrm{mmol})$, or Cat. $(20 \mathrm{~mol} \%)$ in $2 \mathrm{~mL}$ DCM at ambient temperature for $8 \mathrm{~h} .{ }^{b}$ Yields were calculated from the isolated compound 6 a or 9a respectively. ${ }^{c}$ Yields were calculated from the isolated compound 7a or 10a respectively. ${ }^{d}$ ee values were calculated from chiral HPLC analysis of major isomer $6{ }^{e}$ ee values were calculated from chiral HPLC analysis of major isomer $\mathbf{1 0 .}$ 
yields (entries 12-18). At last, we scaled up the reaction to gram synthesis, result proved that $3 \mathrm{a}(3.13 \mathrm{mmol})$ and $\mathbf{4 a}(3.44 \mathrm{mmol})$ were well tolerated in DCM at $50{ }^{\circ} \mathrm{C}$, generating the target compound 10a in 80\% yield and $9: 1 \mathrm{dr}$ (entry 19). The relative configuration of $10 a$ was determined by X-ray crystallographic analysis (Fig. 2), and the relative configurations of other products 10 were tentatively assigned by analogy. ${ }^{\mathbf{1 4}}$

We turned to developing an asymmetric catalytic version of this reaction using chiral sulfur ylides and hydrogen bond catalysts. We screened a variety of chiral sulfur ylides for their ability to generate the chiral product $\mathbf{6 a}$, but unfortunately, the best results we obtained were $61 \%$ yield and $18 \%$ ee (Table 4 ). Adding hydrogen-bonding catalysts improved yield to $77 \%$ and ee to $39 \%$ (entries 7-9). Similarly, we screened chiral sulfur ylides to afford the chiral product 10a, and obtained initial results of $53 \%$ yield and $19 \%$ ee. Screening of hydrogenbonding catalysts identified Cat. 3 as the best, affording product 10a in $72 \%$ yield with $32 \%$ ee (entries $16-18$ ).

\section{Conclusions}

In summary, we set up a protecting group-controlled strategy to regulate ring size via sulfur ylide. This powerful method allows access to structurally important cyclopropane- and dihydrofuran-fused spirooxindoles containing vicinal quaternary carbon centers. This approach exhibits good functional group tolerance as well as excellent regio-, chemo- and diastereoselectivity. It can be scaled up to gram synthesis. Further studies on the bioactivity of promising spirooxindoles will be reported in due course.

\section{Experimental}

\section{General method for the synthesis}

NMR data were obtained for ${ }^{1} \mathrm{H}$ at $400 \mathrm{MHz}$ or $600 \mathrm{MHz}$, and for ${ }^{13} \mathrm{C}$ at $100 \mathrm{MHz}$ or $150 \mathrm{MHz}$. Chemical shifts are reported in ppm from tetramethylsilane with the solvent resonance in $\mathrm{CDCl}_{3}$ solution as the internal standard. ESI HRMS was performed on a Waters SYNAPT G2. Column chromatography was performed on silica gel (400-500 mesh) eluting with ethyl acetate and petroleum ether. TLC was performed on glassbacked silica plates. UV light and $\mathrm{I}_{2}$ were used to visualize products.

\section{General procedure for the synthesis of compounds 6}

To a solution of tetra-substituted oxindole olefins $2(0.15 \mathrm{mmol})$ in $\mathrm{CH}_{2} \mathrm{Cl}_{2}(2.0 \mathrm{~mL})$ was added sulfur ylides $4(0.165 \mathrm{mmol})$ at $25{ }^{\circ} \mathrm{C}$. The reaction mixture was stirred until the reaction completed (monitored by TLC). Then the reaction mixture was concentrated and the residue was purified by flash chromatography on silica gel (petroleum ether/ethyl acetate $=8: 1$ to $5: 1$ ) to give the compounds 6 which were dried under vacuum and further analyzed by ${ }^{1} \mathrm{H}-\mathrm{NMR},{ }^{13} \mathrm{C}-\mathrm{HMR}$, HRMS, etc.

Compound 5a was obtained according to the similar procedure. White solid, $45 \%$ yield (23.4 $\mathrm{mg}$ ). The diastereomeric ratio was determined to be $18: 1$ by crude ${ }^{1} \mathrm{H}-\mathrm{NMR}$ analysis. Mp 165-
$166{ }^{\circ} \mathrm{C} ;{ }^{1} \mathrm{H}$ NMR $\left(400 \mathrm{MHz}, \mathrm{CDCl}_{3}\right) \delta(\mathrm{ppm}) 8.37$ (s, 1H), 7.89 (dd, $J=8.0,0.8 \mathrm{~Hz}, 2 \mathrm{H}), 7.58-7.52(\mathrm{~m}, 1 \mathrm{H}), 7.48-7.39(\mathrm{~m}, 2 \mathrm{H}), 7.22$ $(\mathrm{td}, J=7.6,1.2 \mathrm{~Hz}, 1 \mathrm{H}), 7.03(\mathrm{~d}, J=7.2 \mathrm{~Hz}, 1 \mathrm{H}), 6.96(\mathrm{td}, J=7.6$, $1.2 \mathrm{~Hz}, 1 \mathrm{H}), 6.91(\mathrm{~d}, J=8.0 \mathrm{~Hz}, 1 \mathrm{H}), 4.26(\mathrm{~s}, 1 \mathrm{H}), 2.36(\mathrm{~s}, 3 \mathrm{H})$, 2.26 (s, 3H); ${ }^{13} \mathrm{C}$ NMR (100 MHz, $\left.\mathrm{CDCl}_{3}\right) \delta(\mathrm{ppm})$ 198.0, 196.1, 192.0, 173.5, 141.2, 136.5, 134.1, 128.9, 128.7, 128.5, 126.7, 122.5, 121.1, 110.1, 62.5, 43.6, 42.9, 29.8, 28.4; ESI HRMS: calcd for $\mathrm{C}_{21} \mathrm{H}_{17} \mathrm{NO}_{4} \mathrm{Na}^{+} 370.1055$, found 370.1056.

Compound 6 a was obtained as white solid in $82 \%$ yield (55.0 $\mathrm{mg}$ ). The diastereomeric ratio was determined to be $20: 1$ by crude ${ }^{1} \mathrm{H}-\mathrm{NMR}$ analysis. Mp $170-171{ }^{\circ} \mathrm{C} ;{ }^{1} \mathrm{H}$ NMR $(400 \mathrm{MHz}$, $\left.\mathrm{CDCl}_{3}\right) \delta(\mathrm{ppm}) 7.90(\mathrm{dd}, J=8.0,0.8 \mathrm{~Hz}, 2 \mathrm{H}), 7.85(\mathrm{~d}, J=8.0 \mathrm{~Hz}$, $1 \mathrm{H}), 7.59-7.55(\mathrm{~m}, 1 \mathrm{H}), 7.43(\mathrm{t}, J=7.6 \mathrm{~Hz}, 2 \mathrm{H}), 7.30-7.27(\mathrm{~m}$, 1H), 7.09-7.02 (m, 2H), $4.28(\mathrm{~s}, 1 \mathrm{H}), 2.35(\mathrm{~s}, 3 \mathrm{H}), 2.26(\mathrm{~s}, 3 \mathrm{H})$, 1.64 (s, 9H); ${ }^{13} \mathrm{C}$ NMR (100 MHz, $\left.\mathrm{CDCl}_{3}\right) \delta(\mathrm{ppm})$ 197.7, 195.9, $191.7,170.9$, 148.5, 140.5, 136.4, 134.2, 128.9, 128.6, 126.0, 124.1, 119.8, 114.6, 85.3, 63.0, 44.5, 43.1, 29.8, 28.4, 28.1; ESI HRMS: calcd for $\mathrm{C}_{26} \mathrm{H}_{25} \mathrm{NO}_{6} \mathrm{Na}^{+} 470.1580$, found 470.1581 .

Compound $\mathbf{6 b}$ was obtained as white solid, $74 \%$ yield $(51.7$ $\mathrm{mg}$ ). The diastereomeric ratio was determined to be $18: 1$ by crude ${ }^{1} \mathrm{H}-\mathrm{NMR}$ analysis. Mp $143-144{ }^{\circ} \mathrm{C} ;{ }^{1} \mathrm{H}$ NMR $(600 \mathrm{MHz}$, $\left.\mathrm{CDCl}_{3}\right) \delta(\mathrm{ppm}) 7.88(\mathrm{~d}, J=7.8 \mathrm{~Hz}, 1 \mathrm{H}), 7.75(\mathrm{td}, J=7.2,1.8 \mathrm{~Hz}$, $1 \mathrm{H}), 7.54-7.49(\mathrm{~m}, 1 \mathrm{H}), 7.32(\mathrm{dt}, J=7.8,1.2 \mathrm{~Hz}, 1 \mathrm{H}), 7.21(\mathrm{t}, J=$ $7.2 \mathrm{~Hz}, 1 \mathrm{H}), 7.08-7.03(\mathrm{~m}, 2 \mathrm{H}), 6.93(\mathrm{dd}, J=7.8,1.2 \mathrm{~Hz}, 1 \mathrm{H}), 4.17$ $(\mathrm{d}, J=3.0 \mathrm{~Hz}, 1 \mathrm{H}), 2.37(\mathrm{~s}, 3 \mathrm{H}), 2.29(\mathrm{~s}, 3 \mathrm{H}), 1.65(\mathrm{~s}, 9 \mathrm{H}) ;{ }^{13} \mathrm{C}$ NMR (150 MHz, $\mathrm{CDCl}_{3}$ ) $\delta(\mathrm{ppm})$ 198.0, 195.9, 190.1 (d, $J_{\mathrm{CF}}=3.0$ $\mathrm{Hz}), 170.9,161.9\left(\mathrm{~d}, J_{\mathrm{CF}}=255.0 \mathrm{~Hz}\right), 148.7,140.7,135.8\left(\mathrm{~d}, J_{\mathrm{CF}}=\right.$ $9.0 \mathrm{~Hz}), 130.7\left(\mathrm{~d}, J_{\mathrm{CF}}=1.5 \mathrm{~Hz}\right), 128.9,125.6,124.8\left(\mathrm{~d}, J_{\mathrm{CF}}=4.5\right.$ $\mathrm{Hz}), 124.0,119.9,117.0,116.9,114.7,85.2,63.2,48.6\left(\mathrm{~d}, J_{\mathrm{CF}}=\right.$ $7.5 \mathrm{~Hz}$ ), 43.3 (d, $\left.J_{\mathrm{CF}}=3.0 \mathrm{~Hz}\right), 29.9,28.6$, 28.2; ESI HRMS: calcd for $\mathrm{C}_{26} \mathrm{H}_{24} \mathrm{FNO}_{6} \mathrm{Na}^{+}$488.1485, found 488.1487.

Compound $6 \mathbf{c}$ was obtained as white solid, $70 \%$ yield (48.9 $\mathrm{mg}$ ). The diastereomeric ratio was determined to be $20: 1$ by crude ${ }^{1} \mathrm{H}-\mathrm{NMR}$ analysis. Mp $145-146{ }^{\circ} \mathrm{C} ;{ }^{1} \mathrm{H}$ NMR $(600 \mathrm{MHz}$, $\left.\mathrm{CDCl}_{3}\right) \delta(\mathrm{ppm}) 7.96-7.93(\mathrm{~m}, 2 \mathrm{H}), 7.86(\mathrm{~d}, J=8.2 \mathrm{~Hz}, 1 \mathrm{H}), 7.31$ (dt, $J=9.0,1.8 \mathrm{~Hz}, 1 \mathrm{H}), 7.12-7.09(\mathrm{~m}, 2 \mathrm{H}), 7.07$ (td, $J=7.8$, $1.2 \mathrm{~Hz}, 1 \mathrm{H}), 7.01$ (dd, $J=7.8,1.2 \mathrm{~Hz}, 1 \mathrm{H}), 4.22(\mathrm{~s}, 1 \mathrm{H}), 2.35$ (s, $3 \mathrm{H}), 2.26(\mathrm{~s}, 3 \mathrm{H}), 1.65(\mathrm{~s}, 9 \mathrm{H}) ;{ }^{13} \mathrm{C} \mathrm{NMR}\left(150 \mathrm{MHz}, \mathrm{CDCl}_{3}\right)$ $\delta(\mathrm{ppm}) 197.7,195.9,190.1,171.0,166.4\left(\mathrm{~d}, J_{\mathrm{CF}}=255.0 \mathrm{~Hz}\right)$, 148.6, 140.5, 132.9 (d, $\left.J_{\mathrm{CF}}=4.5 \mathrm{~Hz}\right), 131.4\left(\mathrm{~d}, J_{\mathrm{CF}}=10.5 \mathrm{~Hz}\right)$, 129.1, 126.0, 124.2, 119.7, 116.2 (d, $\left.J_{\mathrm{CF}}=22.5 \mathrm{~Hz}\right), 114.7,85.4$, 63.1, 44.3, 43.1, 29.9, 28.5, 28.1; ESI HRMS: calcd for $\mathrm{C}_{26} \mathrm{H}_{24}$ $\mathrm{FNO}_{6} \mathrm{Na}^{+} 488.1485$, found 488.1487 .

Compound $6 \mathbf{d}$ was obtained as yellow solid, $63 \%$ yield ( 48.8 $\mathrm{mg}$ ). The diastereomeric ratio was determined to be $20: 1$ by crude ${ }^{1} \mathrm{H}-\mathrm{NMR}$ analysis. Mp $144-145{ }^{\circ} \mathrm{C} ;{ }^{1} \mathrm{H}$ NMR $(400 \mathrm{MHz}$, $\left.\mathrm{CDCl}_{3}\right) \delta(\mathrm{ppm}) 8.01(\mathrm{~d}, J=2.0 \mathrm{~Hz}, 1 \mathrm{H}), 7.88(\mathrm{~d}, J=8.0 \mathrm{~Hz}, 1 \mathrm{H})$, $7.75(\mathrm{dd}, J=8.4,2.4 \mathrm{~Hz}, 1 \mathrm{H}), 7.52(\mathrm{~d}, J=8.4 \mathrm{~Hz}, 1 \mathrm{H}), 7.33(\mathrm{td}, J=$ 8.8, $1.6 \mathrm{~Hz}, 1 \mathrm{H}), 7.08$ (td, $J=8.0,1.2 \mathrm{~Hz}, 1 \mathrm{H}), 6.98$ (dd. $J=8.0$, $1.2 \mathrm{~Hz}, 1 \mathrm{H}), 4.13(\mathrm{~s}, 1 \mathrm{H}), 2.32(\mathrm{~s}, 3 \mathrm{H}), 2.28(\mathrm{~s}, 3 \mathrm{H}), 1.65(\mathrm{~s}, 9 \mathrm{H})$; ${ }^{13} \mathrm{C}$ NMR $\left(100 \mathrm{MHz}, \mathrm{CDCl}_{3}\right) \delta(\mathrm{ppm})$ 197.5, 195.6, 189.7, 170.7, $148.4,140.5$, 139.0, 135.9, 133.8, 131.0, 130.3, 129.2, 127.5, 125.8, 124.2, 119.4, 114.8, 85.4, 63.3, 43.8, 43.3, 29.8, 28.5, 28.1; ESI HRMS: calcd for $\mathrm{C}_{26} \mathrm{H}_{23} \mathrm{Cl}_{2} \mathrm{NO}_{6} \mathrm{Na}^{+}$538.0800, found 538.0802 .

Compound 6e was obtained as yellow solid, 75\% yield $(59.2$ $\mathrm{mg}$ ). The diastereomeric ratio was determined to be $18: 1$ by 
crude ${ }^{1} \mathrm{H}$-NMR analysis. Mp $147-148{ }^{\circ} \mathrm{C} ;{ }^{1} \mathrm{H}$ NMR $(400 \mathrm{MHz}$, $\left.\mathrm{CDCl}_{3}\right) \delta(\mathrm{ppm}) 7.86(\mathrm{~d}, J=8.0 \mathrm{~Hz}, 1 \mathrm{H}), 7.77(\mathrm{~d}, J=8.4 \mathrm{~Hz}, 2 \mathrm{H})$, $7.57(\mathrm{~d}, J=8.4 \mathrm{~Hz}, 2 \mathrm{H}), 7.31(\mathrm{t}, J=7.6 \mathrm{~Hz}, 1 \mathrm{H}), 7.07(\mathrm{t}, J=8.0 \mathrm{~Hz}$, $1 \mathrm{H}), 6.98(\mathrm{~d}, J=8.0 \mathrm{~Hz}, 1 \mathrm{H}), 4.20(\mathrm{~s}, 1 \mathrm{H}), 2.34(\mathrm{~s}, 3 \mathrm{H}), 2.26(\mathrm{~s}$, 3H), 1.65 (s, 9H); ${ }^{13} \mathrm{C}$ NMR (150 MHz, $\left.\mathrm{CDCl}_{3}\right) \delta(\mathrm{ppm})$ 197.5, $195.7,190.7,170.8,148.4,140.4$, 135.0, 132.2, 129.9, 129.0, 125.8, 124.0, 119.5, 114.7, 85.32, 63.0, 44.1, 43.0, 29.8, 28.4, 28.0; ESI HRMS: calcd for $\mathrm{C}_{26} \mathrm{H}_{24} \mathrm{BrNO}_{6} \mathrm{Na}^{+}$548.0685, found 548.0686 .

Compound $\mathbf{6 f}$ was obtained as white solid, $80 \%$ yield (55.4 $\mathrm{mg}$ ). The diastereomeric ratio was determined to be $20: 1$ by crude ${ }^{1} \mathrm{H}-\mathrm{NMR}$ analysis. Mp $142-143{ }^{\circ} \mathrm{C} ;{ }^{1} \mathrm{H}$ NMR $(600 \mathrm{MHz}$, $\left.\mathrm{CDCl}_{3}\right) \delta(\mathrm{ppm}) 7.88(\mathrm{~d}, J=7.8 \mathrm{~Hz}, 1 \mathrm{H}), 7.56(\mathrm{~d}, J=7.2 \mathrm{~Hz}, 1 \mathrm{H})$, $7.38(\mathrm{td}, J=7.8,1.2 \mathrm{~Hz}, 1 \mathrm{H}), 7.34-7.31(\mathrm{~m}, 1 \mathrm{H}), 7.22(\mathrm{dd}, J=$ 15.6, 9.0 Hz, 2H), 7.09 (dd, $J=6.0,2.4 \mathrm{~Hz}, 2 \mathrm{H}), 4.12(\mathrm{~s}, 1 \mathrm{H}), 2.42$ (s, 3H), $2.36(\mathrm{~s}, 3 \mathrm{H}), 2.25(\mathrm{~s}, 3 \mathrm{H}), 1.64(\mathrm{~s}, 9 \mathrm{H}) ;{ }^{13} \mathrm{C}$ NMR $(150$ $\left.\mathrm{MHz}, \mathrm{CDCl}_{3}\right) \delta(\mathrm{ppm}) 195.9,194.7,170.9,148.5,140.5,132.6$, 132.1, 129.5, 128.9, 126.2, 126.1, 124.0, 119.8, 114.6, 85.3, 63.2, 47.3, 43.5, 29.8, 28.4, 28.1, 21.2; ESI HRMS: calcd for $\mathrm{C}_{27} \mathrm{H}_{27} \mathrm{NO}_{6} \mathrm{Na}^{+}$484.1736, found 484.1734.

Compound $6 \mathrm{~g}$ was obtained as white solid, $81 \%$ yield (58.0 $\mathrm{mg}$ ). The diastereomeric ratio was determined to be $20: 1$ by crude ${ }^{1} \mathrm{H}$-NMR analysis. Mp $145-146{ }^{\circ} \mathrm{C} ;{ }^{1} \mathrm{H}$ NMR $(400 \mathrm{MHz}$, $\left.\mathrm{CDCl}_{3}\right) \delta(\mathrm{ppm}) 7.88(\mathrm{~d}, J=8.8 \mathrm{~Hz}, 2 \mathrm{H}), 7.84(\mathrm{~d}, J=8.4 \mathrm{~Hz}, 1 \mathrm{H})$, 7.30-7.26 (m, 1H), 7.06-7.04 (m, 2H), 6.88 (d, J=8.8 Hz, 2H), 4.25 (s, 1H), 3.83 (s, 3H), $2.36(\mathrm{~s}, 3 \mathrm{H}), 2.25$ (s, 3H), $1.64(\mathrm{~s}, 9 \mathrm{H})$; ${ }^{13} \mathrm{C}$ NMR (100 MHz, $\left.\mathrm{CDCl}_{3}\right) \delta(\mathrm{ppm})$ 197.9, 196.0, 189.7, 171.1, $164.5,148.6,140.4,131.1,129.5,128.7,126.1,124.0,120.0$, 114.5, 114.1, 85.2, 62.8, 55.6, 44.6, 42.8, 29.9, 28.3, 28.1; ESI HRMS: calcd for $\mathrm{C}_{27} \mathrm{H}_{27} \mathrm{NO}_{7} \mathrm{H}^{+} 500.1685$, found 500.1688 .

Compound $6 \mathrm{~h}$ was obtained as white solid, $68 \%$ yield (46.3 $\mathrm{mg}$ ). The diastereomeric ratio was determined to be $20: 1$ by crude ${ }^{1} \mathrm{H}-\mathrm{NMR}$ analysis. Mp $137-138{ }^{\circ} \mathrm{C} ;{ }^{1} \mathrm{H}$ NMR $(600 \mathrm{MHz}$, $\left.\mathrm{CDCl}_{3}\right) \delta(\mathrm{ppm}) 7.86(\mathrm{~d}, J=7.8 \mathrm{~Hz}, 1 \mathrm{H}), 7.81(\mathrm{~d}, J=3.0 \mathrm{~Hz}, 1 \mathrm{H})$, 7.69 (d, $J=4.8 \mathrm{~Hz}, 1 \mathrm{H}), 7.34-7.31(\mathrm{~m}, 1 \mathrm{H}), 7.14-7.10(\mathrm{~m}, 3 \mathrm{H})$, 4.15 (s, 1H), 2.35 (s, 3H), 2.25 (s, 3H), 1.64 (s, 9H); ${ }^{13} \mathrm{C}$ NMR (150 $\left.\mathrm{MHz}, \mathrm{CDCl}_{3}\right) \delta(\mathrm{ppm})$ 197.5, 195.6, 183.9, 170.9, 148.6, 143.7, 140.6, 135.9, 134.1, 129.0, 128.9, 126.5, 124.2, 119.8, 114.7, 85.4, 62.9, 60.5, 45.0, 43.2, 29.8, 28.1; ESI HRMS: calcd for $\mathrm{C}_{24} \mathrm{H}_{23}$ $\mathrm{NO}_{6} \mathrm{SNa}^{+} 476.1144$, found 476.1145 .

Compound $6 \mathbf{i}$ was obtained as white solid, $65 \%$ yield $(48.5$ $\mathrm{mg}$ ). The diastereomeric ratio was determined to be $10: 1$ by crude ${ }^{1} \mathrm{H}-\mathrm{NMR}$ analysis. Mp $153-154{ }^{\circ} \mathrm{C} ;{ }^{1} \mathrm{H}$ NMR $(400 \mathrm{MHz}$, $\left.\mathrm{CDCl}_{3}\right) \delta(\mathrm{ppm}) 8.47(\mathrm{~s}, 1 \mathrm{H}), 7.97-7.92(\mathrm{~m}, 2 \mathrm{H}), 7.85-7.82(\mathrm{~m}$, $3 \mathrm{H}), 7.60-7.55(\mathrm{~m}, 2 \mathrm{H}), 7.30-7.26(\mathrm{~m}, 1 \mathrm{H}), 7.13-7.05(\mathrm{~m}, 2 \mathrm{H})$, $4.46(\mathrm{~s}, 1 \mathrm{H}), 2.38(\mathrm{~s}, 3 \mathrm{H}), 2.30(\mathrm{~s}, 3 \mathrm{H}), 1.65(\mathrm{~s}, 9 \mathrm{H}) ;{ }^{13} \mathrm{C}$ NMR $(100$ $\left.\mathrm{MHz}, \mathrm{CDCl}_{3}\right) \delta(\mathrm{ppm}) 197.8,195.9,191.4,171.1,148.5,140.5$, $135.9,133.8,132.3,130.9,129.9,129.3,128.9,128.8,127.8$, 127.2 , 126.2, 124.1, 123.5, 119.8, 114.6, 85.3, 63.1, 44.6, 43.3, 29.9, 28.4, 28.1; ESI HRMS: calcd for $\mathrm{C}_{30} \mathrm{H}_{27} \mathrm{NO}_{6} \mathrm{Na}^{+} 520.1736$, found 520.1738 .

Compound $\mathbf{6 j}$ was obtained as white solid, $79 \%$ yield $(49.2$ $\mathrm{mg}$ ). The diastereomeric ratio was determined to be $20: 1$ by crude ${ }^{1} \mathrm{H}-\mathrm{NMR}$ analysis. Mp $149-150{ }^{\circ} \mathrm{C} ;{ }^{1} \mathrm{H}$ NMR $(600 \mathrm{MHz}$, $\left.\mathrm{CDCl}_{3}\right) \delta(\mathrm{ppm}) 7.91(\mathrm{~d}, J=8.4 \mathrm{~Hz}, 1 \mathrm{H}), 7.36(\mathrm{dt}, J=8.4,2.4 \mathrm{~Hz}$, $1 \mathrm{H}), 7.14-7.12(\mathrm{~m}, 2 \mathrm{H}), 4.21-4.18(\mathrm{~m}, 1 \mathrm{H}), 4.14-4.11(\mathrm{~m}, 1 \mathrm{H})$, $3.33(\mathrm{~s}, 1 \mathrm{H}), 2.30(\mathrm{~s}, 3 \mathrm{H}), 2.27(\mathrm{~s}, 3 \mathrm{H}), 1.63(\mathrm{~s}, 9 \mathrm{H}), 1.22(\mathrm{t}, J=$
$7.2 \mathrm{~Hz}, 3 \mathrm{H}) ;{ }^{13} \mathrm{C} \mathrm{NMR}\left(150 \mathrm{MHz}, \mathrm{CDCl}_{3}\right) \delta(\mathrm{ppm})$ 197.4, 195.5, 170.6, 166.0, 148.6, 140.8, 129.1, 125.9, 124.0, 119.9, 114.8, 85.3, 62.2, 62.1, 41.6, 40.5, 29.6, 28.6, 28.1, 14.13; ESI HRMS: calcd for $\mathrm{C}_{22} \mathrm{H}_{25} \mathrm{NO}_{7} \mathrm{Na}^{+}$438.1529, found 438.1531.

Compound 6k was obtained as white solid, 68\% yield (47.5 $\mathrm{mg})$. The diastereomeric ratio was determined to be $13: 1$ by crude ${ }^{1} \mathrm{H}$-NMR analysis. Mp $172-173{ }^{\circ} \mathrm{C} ;{ }^{1} \mathrm{H}$ NMR $(600 \mathrm{MHz}$, $\left.\mathrm{CDCl}_{3}\right) \delta(\mathrm{ppm}) 7.92(\mathrm{dd}, J=8.4,1.2 \mathrm{~Hz}, 2 \mathrm{H}), 7.84(\mathrm{dd}, J=9.0$, $4.8 \mathrm{~Hz}, 1 \mathrm{H}), 7.61-7.58(\mathrm{~m}, 1 \mathrm{H}), 7.46(\mathrm{dd}, J=7.8,1.8 \mathrm{~Hz}, 2 \mathrm{H}), 7.00$ $(\mathrm{td}, J=9.0,3.0 \mathrm{~Hz}, 1 \mathrm{H}), 6.82(\mathrm{dd}, J=9.6,3.0 \mathrm{~Hz}, 1 \mathrm{H}), 4.30(\mathrm{~s}$, 1H), 2.35 (s, 3H), 2.26 (s, 3H), $1.64(\mathrm{~s}, 9 \mathrm{H}) ;{ }^{13} \mathrm{C}$ NMR $(150 \mathrm{MHz}$, $\left.\mathrm{CDCl}_{3}\right) \delta(\mathrm{ppm}) 197.4,195.8,191.5,170.7,159.3\left(\mathrm{~d}, J_{\mathrm{CF}}=240\right.$ $\mathrm{Hz}), 148.5,136.4\left(\mathrm{~d}, J_{\mathrm{CF}}=36 \mathrm{~Hz}\right), 134.5,129.1,128.7,121.7$ (d, $\left.J_{\mathrm{CF}}=10.5 \mathrm{~Hz}\right), 115.7\left(\mathrm{~d}, J_{\mathrm{CF}}=9.0 \mathrm{~Hz}\right), 115.6,114.1,113.9,85.6$, 63.2, 44.7, 43.0, 29.8, 28.4, 28.1; ESI HRMS: calcd for $\mathrm{C}_{26} \mathrm{H}_{24^{-}}$ $\mathrm{FNO}_{6} \mathrm{Na}^{+} 488.1485$, found 488.1483 .

Compound 61 was obtained as white solid, $70 \%$ yield $(48.9$ $\mathrm{mg}$ ). The diastereomeric ratio was determined to be $20: 1$ by crude ${ }^{1} \mathrm{H}-\mathrm{NMR}$ analysis. Mp 157-158 ${ }^{\circ} \mathrm{C} ;{ }^{1} \mathrm{H}$ NMR $(600 \mathrm{MHz}$, $\left.\mathrm{CDCl}_{3}\right) \delta(\mathrm{ppm}) 7.90(\mathrm{~d}, J=7.2 \mathrm{~Hz}, 2 \mathrm{H}), 7.60-7.58(\mathrm{~m}, 1 \mathrm{H}), 7.45$ $(\mathrm{t}, J=7.8 \mathrm{~Hz}, 2 \mathrm{H}), 7.07-7.01(\mathrm{~m}, 2 \mathrm{H}), 6.84(\mathrm{dd}, J=7.8,1.2 \mathrm{~Hz}$, $1 \mathrm{H}), 4.30(\mathrm{~s}, 1 \mathrm{H}), 2.35(\mathrm{~s}, 3 \mathrm{H}), 2.27(\mathrm{~s}, 3 \mathrm{H}), 1.61(\mathrm{~s}, 9 \mathrm{H}) ;{ }^{13} \mathrm{C} \mathrm{NMR}$ $\left(150 \mathrm{MHz}, \mathrm{CDCl}_{3}\right) \delta(\mathrm{ppm})$ 197.2, 195.8, 191.5, 170.4, 148.4 (d, $\left.J_{\mathrm{CF}}=249.1 \mathrm{~Hz}\right), 147.0,136.3,134.5,129.1,128.7,127.6\left(\mathrm{~d}, J_{\mathrm{CF}}=\right.$ $10.4 \mathrm{~Hz}), 124.9\left(\mathrm{~d}, J_{\mathrm{CF}}=7.4 \mathrm{~Hz}\right), 122.9\left(\mathrm{~d}, J_{\mathrm{CF}}=2.2 \mathrm{~Hz}\right), 122.1(\mathrm{~d}$, $\left.J_{\mathrm{CF}}=4.0 \mathrm{~Hz}\right), 117.1\left(\mathrm{~d}, J_{\mathrm{CF}}=20.0 \mathrm{~Hz}\right), 86.0,63.2,44.5,43.0,29.9$, 28.4, 27.7; ESI HRMS: calcd for $\mathrm{C}_{26} \mathrm{H}_{24} \mathrm{FNO}_{6} \mathrm{Na}^{+} 488.1485$, found 488.1483 .

Compound $6 \mathrm{~m}$ was obtained as yellow solid, $72 \%$ yield (41.9 $\mathrm{mg}$ ). The diastereomeric ratio was determined to be $20: 1$ by crude ${ }^{1} \mathrm{H}-\mathrm{NMR}$ analysis. Mp $136-137{ }^{\circ} \mathrm{C} ;{ }^{1} \mathrm{H}$ NMR $(400 \mathrm{MHz}$, $\left.\mathrm{CDCl}_{3}\right) \delta(\mathrm{ppm}) 7.93(\mathrm{~d}, J=7.6 \mathrm{~Hz}, 2 \mathrm{H}), 7.82(\mathrm{~d}, J=8.8 \mathrm{~Hz}, 1 \mathrm{H})$, $7.60(\mathrm{t}, J=7.2 \mathrm{~Hz}, 1 \mathrm{H}), 7.46(\mathrm{t}, J=7.6 \mathrm{~Hz}, 2 \mathrm{H}), 7.29(\mathrm{~d}, J=1.2 \mathrm{~Hz}$, $1 \mathrm{H}), 7.05(\mathrm{~s}, 1 \mathrm{H}), 4.29(\mathrm{~s}, 1 \mathrm{H}), 2.34(\mathrm{~s}, 3 \mathrm{H}), 2.26(\mathrm{~s}, 3 \mathrm{H}), 1.64(\mathrm{~s}$, $9 \mathrm{H}) ;{ }^{13} \mathrm{C}$ NMR (150 MHz, $\mathrm{CDCl}_{3}$ ) $\delta$ (ppm) 197.2, 195.2, 191.3, 170.3 , 148.3, 138.9, 136.2, 134.4, 129.7, 128.9, 128.8, 128.6, 126.2, 121.5, 115.6, 85.6, 63.1, 44.6, 42.7, 29.6, 28.3, 28.0; ESI HRMS: calcd for $\mathrm{C}_{26} \mathrm{H}_{24} \mathrm{ClNO}_{6} \mathrm{Na}^{+} 504.1190$, found 504.1189.

Compound 6n was obtained as yellow solid, $79 \%$ yield (57.1 $\mathrm{mg}$ ). The diastereomeric ratio was determined to be $20: 1$ by crude ${ }^{1} \mathrm{H}-\mathrm{NMR}$ analysis. Mp $145-146{ }^{\circ} \mathrm{C} ;{ }^{1} \mathrm{H}$ NMR $(400 \mathrm{MHz}$, $\left.\mathrm{CDCl}_{3}\right) \delta(\mathrm{ppm}) 7.93(\mathrm{~d}, J=2.0 \mathrm{~Hz}, 1 \mathrm{H}), 7.90(\mathrm{~d}, J=7.6 \mathrm{~Hz}, 2 \mathrm{H})$, $7.59(\mathrm{t}, J=7.6 \mathrm{~Hz}, 1 \mathrm{H}), 7.45(\mathrm{t}, J=8.0 \mathrm{~Hz}, 2 \mathrm{H}), 7.05(\mathrm{dd}, J=8.4$, $1.6 \mathrm{~Hz}, 1 \mathrm{H}), 6.96(\mathrm{~d}, J=8.8 \mathrm{~Hz}, 1 \mathrm{H}), 4.28(\mathrm{~s}, 1 \mathrm{H}), 2.34(\mathrm{~s}, 3 \mathrm{H})$, $2.25(\mathrm{~s}, 3 \mathrm{H}), 1.64(\mathrm{~s}, 9 \mathrm{H}) ;{ }^{13} \mathrm{C} \mathrm{NMR}\left(100 \mathrm{MHz}, \mathrm{CDCl}_{3}\right) \delta(\mathrm{ppm})$ 197.3, 195.8, 191.6, 170.5, 148.3, 141.3, 136.3, 135.0, 134.4, 129.0, 128.6, 127.0, 124.2, 118.2, 115.4, 85.8, 62.9, 44.6, 42.8, 29.8, 28.3, 28.0; ESI HRMS: calcd for $\mathrm{C}_{26} \mathrm{H}_{24} \mathrm{ClNO}_{6} \mathrm{Na}^{+}$504.1190, found 504.1191.

Compound 60 was obtained as yellow solid, $80 \%$ yield $(63.2$ $\mathrm{mg}$ ). The diastereomeric ratio was determined to be $15: 1$ by crude ${ }^{1} \mathrm{H}-\mathrm{NMR}$ analysis. Mp $142-143{ }^{\circ} \mathrm{C} ;{ }^{1} \mathrm{H}$ NMR $(600 \mathrm{MHz}$, $\left.\mathrm{CDCl}_{3}\right) \delta(\mathrm{ppm}) 7.93(\mathrm{~d}, J=7.2 \mathrm{~Hz}, 2 \mathrm{H}), 7.76(\mathrm{~d}, J=9.0 \mathrm{~Hz}, 1 \mathrm{H})$, $7.60(\mathrm{t}, J=7.8 \mathrm{~Hz}, 1 \mathrm{H}), 7.446(\mathrm{t}, J=7.8 \mathrm{~Hz}, 2 \mathrm{H}), 7.43(\mathrm{dd}, J=9.0$, $1.8 \mathrm{~Hz}, 1 \mathrm{H}), 7.19$ (d, J=1.8 Hz, 1H), $4.28(\mathrm{~s}, 1 \mathrm{H}), 2.34(\mathrm{~s}, 3 \mathrm{H})$, $2.26(\mathrm{~s}, 3 \mathrm{H}), 1.63(\mathrm{~s}, 9 \mathrm{H}) ;{ }^{13} \mathrm{C} \mathrm{NMR}\left(150 \mathrm{MHz}, \mathrm{CDCl}_{3}\right) \delta(\mathrm{ppm})$ 197.2 , 195.5, 191.3, 170.1, 148.2, 139.4, 136.2, 134.4, 131.8, 
129.0, 128.9, 128.6, 121.8, 117.3, 115.9, 85.6, 63.2, 44.6, 42.6, 29.6, 28.3, 27.9; ESI HRMS: calcd for $\mathrm{C}_{26} \mathrm{H}_{24} \mathrm{BrNO}_{6} \mathrm{Na}^{+} 548.0685$, found 548.0687 .

Compound 6p was obtained as yellow solid, $81 \%$ yield (63.9 $\mathrm{mg}$ ). The diastereomeric ratio was determined to be $9: 1$ by crude ${ }^{1} \mathrm{H}$-NMR analysis. Mp 149-150 ${ }^{\circ} \mathrm{C} ;{ }^{1} \mathrm{H}$ NMR (400 $\mathrm{MHz}, \mathrm{CDCl}_{3}$ ) $\delta(\mathrm{ppm}) 8.09(\mathrm{~d}, J=0.8 \mathrm{~Hz}, 1 \mathrm{H}), 7.90(\mathrm{~d}, J=4.8 \mathrm{~Hz}, 2 \mathrm{H}), 7.61-7.58$ $(\mathrm{m}, 1 \mathrm{H}), 7.45(\mathrm{t}, J=5.2 \mathrm{~Hz}, 2 \mathrm{H}), 7.20(\mathrm{dd}, J=5.6,0.8 \mathrm{~Hz}, 1 \mathrm{H}), 6.90$ $(\mathrm{d}, J=5.6 \mathrm{~Hz}, 1 \mathrm{H}), 4.29(\mathrm{~s}, 1 \mathrm{H}), 2.34(\mathrm{~s}, 3 \mathrm{H}), 2.25(\mathrm{~s}, 3 \mathrm{H}), 1.64(\mathrm{~s}$, 9H); ${ }^{13} \mathrm{C}$ NMR (100 MHz, $\left.\mathrm{CDCl}_{3}\right) \delta$ (ppm) 197.3, 195.9, 191.6, 170.5, 148.3, 141.4, 136.3, 134.5, 129.1, 128.7, 127.3, 127.2, 123.1, 118.8, 118.3, 85.9, 63.0, 44.6, 29.9, 28.4, 28.1, 28.0; ESI HRMS: calcd for $\mathrm{C}_{26} \mathrm{H}_{24} \mathrm{BrNO}_{6} \mathrm{Na}^{+}$548.0685, found 548.0683.

Compound $6 \mathrm{q}$ was obtained as white solid, $85 \%$ yield (58.8 $\mathrm{mg}$ ). The diastereomeric ratio was determined to be $15: 1$ by crude ${ }^{1} \mathrm{H}$-NMR analysis. Mp 168-169 ${ }^{\circ} \mathrm{C}$; ${ }^{1} \mathrm{H}$ NMR $(400 \mathrm{MHz}$, $\left.\mathrm{CDCl}_{3}\right) \delta(\mathrm{ppm}) 7.90(\mathrm{~d}, J=7.2 \mathrm{~Hz}, 2 \mathrm{H}), 7.72(\mathrm{~d}, J=8.4 \mathrm{~Hz}, 1 \mathrm{H})$, 7.59-7.55 (m, 1H), $7.44(\mathrm{t}, J=8.0 \mathrm{~Hz}, 2 \mathrm{H}), 7.10(\mathrm{dd}, J=8.4,0.8 \mathrm{~Hz}$, $1 \mathrm{H}), 6.84(\mathrm{~s}, 1 \mathrm{H}), 4.25(\mathrm{~s}, 1 \mathrm{H}), 2.35(\mathrm{~s}, 3 \mathrm{H}), 2.29(\mathrm{~s}, 3 \mathrm{H}), 2.26(\mathrm{~s}, 3 \mathrm{H})$, 1.64 (s, 9H); ${ }^{13} \mathrm{C}$ NMR (100 MHz, $\left.\mathrm{CDCl}_{3}\right) \delta(\mathrm{ppm})$ 197.8, 195.9, 191.7, 171.1, 148.6, 138.2, 136.5, 134.2, 133.7, 129.5, 128.9, 128.6, 126.5, 119.7, 114.4, 85.1, 63.0, 44.5, 43.2, 29.8, 28.4, 28.1, 21.0; ESI HRMS: calcd for $\mathrm{C}_{27} \mathrm{H}_{27} \mathrm{NO}_{6} \mathrm{Na}^{+}$484.1736, found 484.1740.

Compound 6r was obtained as white solid, $90 \%$ yield $(64.5$ $\mathrm{mg}$ ). The diastereomeric ratio was determined to be $18: 1$ by crude ${ }^{1} \mathrm{H}-\mathrm{NMR}$ analysis. Mp $147-148{ }^{\circ} \mathrm{C} ;{ }^{1} \mathrm{H}$ NMR $(600 \mathrm{MHz}$, $\left.\mathrm{CDCl}_{3}\right) \delta(\mathrm{ppm}) 8.10(\mathrm{dd}, J=8.4,1.2 \mathrm{~Hz}, 2 \mathrm{H}), 7.90(\mathrm{~d}, J=7.8 \mathrm{~Hz}$, 1H), 7.59-7.56 (m, 1H), 7.47 (dt, $J=7.2,1.2 \mathrm{~Hz}, 2 \mathrm{H}), 7.34-7.31$ $(\mathrm{m}, 1 \mathrm{H}), 7.08(\mathrm{dt}, J=7.8,1.8 \mathrm{~Hz}, 1 \mathrm{H}), 7.01(\mathrm{dd}, J=7.8,1.2 \mathrm{~Hz}$, $1 \mathrm{H}), 4.43-4.39(\mathrm{~m}, 1 \mathrm{H}), 4.32-4.29(\mathrm{~m}, 1 \mathrm{H}), 4.10(\mathrm{~s}, 1 \mathrm{H}), 2.29(\mathrm{~s}$, $3 \mathrm{H}), 1.65$ (s, 9H), 1.35 (t, $J=7.2 \mathrm{~Hz}, 3 \mathrm{H}) ;{ }^{13} \mathrm{C}$ NMR $(150 \mathrm{MHz}$, $\left.\mathrm{CDCl}_{3}\right) \delta(\mathrm{ppm})$ 195.3, 191.0, 170.8, 165.5, 148.7, 140.7, 136.6, 133.9, 129.1, 128.9, 128.5, 125.7, 124.1, 119.7, 114.8, 85.0, 63.2, 56.7, 42.1, 42.0, 29.7, 28.2, 14.1; ESI HRMS: calcd for $\mathrm{C}_{27} \mathrm{H}_{27} \mathrm{NO}_{7} \mathrm{Na}^{+} 500.1685$, found 500.1687.

\section{General procedure for the synthesis of compounds 10}

To a solution of Bn-protected tetra-substituted oxindole olefins $3(0.15 \mathrm{mmol})$ in $\mathrm{CH}_{2} \mathrm{Cl}_{2}(2.0 \mathrm{~mL})$ was added sulfur ylides 4 $(0.165 \mathrm{mmol})$ at $50{ }^{\circ} \mathrm{C}$. The reaction mixture was stirred until the reaction completed (monitored by TLC). Then the reaction mixture was concentrated and the residue was purified by flash chromatography on silica gel to give the compounds 10 which were dried under vacuum and further analyzed by ${ }^{1} \mathrm{H}-\mathrm{NMR},{ }^{13} \mathrm{C}$ HMR, HRMS, etc.

Compound 8a was obtained according to the similar procedure. White solid, $48 \%$ yield $(25.0 \mathrm{mg})$. The diastereomeric ratio was determined to be $10: 1$ by crude ${ }^{1} \mathrm{H}-\mathrm{NMR}$ analysis. Mp 164$165{ }^{\circ} \mathrm{C} ;{ }^{1} \mathrm{H}$ NMR $\left(400 \mathrm{MHz}, \mathrm{CDCl}_{3}\right) \delta(\mathrm{ppm}) 7.84(\mathrm{~s}, 1 \mathrm{H}), 7.43-$ $7.38(\mathrm{~m}, 1 \mathrm{H}), 7.35$ (dd, $J=8.4,1.2 \mathrm{~Hz}, 2 \mathrm{H}), 7.22(\mathrm{td}, J=7.6$, $1.6 \mathrm{~Hz}, 2 \mathrm{H}), 6.99-6.95(\mathrm{~m}, 1 \mathrm{H}), 6.91-6.86(\mathrm{~m}, 2 \mathrm{H}), 6.40(\mathrm{~d}, J=$ $7.6 \mathrm{~Hz}, 1 \mathrm{H}), 6.27$ (s, 1H), 2.55 (s, 3H), 2.15 (s, 3H); ${ }^{13} \mathrm{C}$ NMR $(100$ $\left.\mathrm{MHz}, \mathrm{CDCl}_{3}\right) \delta(\mathrm{ppm}) 192.8,191.3,178.8,170.2,139.9,134.8$, 133.6, 129.0, 128.4, 127.5, 127.4, 124.9, 122.8, 118.3, 109.3, 88.8, 62.2, 28.9, 15.8; ESI HRMS: calcd for $\mathrm{C}_{21} \mathrm{H}_{17} \mathrm{NO}_{4} \mathrm{Na}^{+} 370.1055$, found 370.1054 .
Compound 10a was obtained as white solid in $74 \%$ yield (48.6 $\mathrm{mg}$ ). The diastereomeric ratio was determined to be $10: 1$ by crude ${ }^{1} \mathrm{H}-\mathrm{NMR}$ analysis. Mp $168-169{ }^{\circ} \mathrm{C} ;{ }^{1} \mathrm{H}$ NMR $(400 \mathrm{MHz}$, $\left.\mathrm{CDCl}_{3}\right) \delta(\mathrm{ppm}) 7.42(\mathrm{t}, J=7.2 \mathrm{~Hz}, 1 \mathrm{H}), 7.30(\mathrm{~d}, J=4.4 \mathrm{~Hz}, 4 \mathrm{H})$, $7.25(\mathrm{~d}, J=4.8 \mathrm{~Hz}, 3 \mathrm{H}), 7.16(\mathrm{t}, J=7.6 \mathrm{~Hz}, 2 \mathrm{H}), 6.93-6.89(\mathrm{~m}, 2 \mathrm{H})$, $6.86(\mathrm{t}, J=7.2 \mathrm{~Hz}, 1 \mathrm{H}), 6.30(\mathrm{~s}, 1 \mathrm{H}), 6.17(\mathrm{~d}, J=7.6 \mathrm{~Hz}, 1 \mathrm{H}), 5.08$ $(\mathrm{d}, J=15.6 \mathrm{~Hz}, 1 \mathrm{H}), 4.20(\mathrm{~d}, J=16.0 \mathrm{~Hz}, 1 \mathrm{H}), 2.56(\mathrm{~s}, 3 \mathrm{H}), 2.15$ (s, $3 \mathrm{H}) ;{ }^{13} \mathrm{C}$ NMR (100 MHz, $\mathrm{CDCl}_{3}$ ) $\delta$ (ppm) 192.9, 191.0, 177.3, 169.9, 142.2, 135.3, 135.0, 133.4, 128.9, 128.7, 128.3, 127.5, 127.3, 126.9, 124.7, 122.8, 118.4, 108.8, 89.0, 62.0, 44.6, 29.0, 15.9; ESI HRMS: calcd for $\mathrm{C}_{28} \mathrm{H}_{23} \mathrm{NO}_{4} \mathrm{Na}^{+} 460.1525$, found 460.1526 .

Compound 10b was obtained as white solid, $66 \%$ yield (45.1 $\mathrm{mg}$ ). The diastereomeric ratio was determined to be $16: 1$ by crude ${ }^{1} \mathrm{H}-\mathrm{NMR}$ analysis. Mp 154-155 ${ }^{\circ} \mathrm{C} ;{ }^{1} \mathrm{H}$ NMR $(600 \mathrm{MHz}$, $\left.\mathrm{CDCl}_{3}\right) \delta(\mathrm{ppm}) 7.35-7.31(\mathrm{~m}, 4 \mathrm{H}), 7.31-7.24(\mathrm{~m}, 3 \mathrm{H}), 6.95(\mathrm{tt}, J=$ $7.2,1.8 \mathrm{~Hz}, 2 \mathrm{H}), 6.90(\mathrm{t}, J=6.6 \mathrm{~Hz}, 2 \mathrm{H}), 6.86(\mathrm{t}, J=7.8 \mathrm{~Hz}, 1 \mathrm{H})$, 6.83-6.79 (m, 1H), $6.26(\mathrm{~s}, 1 \mathrm{H}), 5.02(\mathrm{~d}, J=15.6 \mathrm{~Hz}, 1 \mathrm{H}), 4.33(\mathrm{~d}$, $J=15.6 \mathrm{~Hz}, 1 \mathrm{H}), 2.55$ (s, 3H), $2.13(\mathrm{~s}, 3 \mathrm{H}) ;{ }^{13} \mathrm{C} \mathrm{NMR}(150 \mathrm{MHz}$, $\left.\mathrm{CDCl}_{3}\right) \delta(\mathrm{ppm}) 191.3\left(\mathrm{~d}, J_{\mathrm{CF}}=4.5 \mathrm{~Hz}\right), 191.0,177.1,169.9,160.4$ $\left(\mathrm{d}, J_{\mathrm{CF}}=253.5 \mathrm{~Hz}\right), 142.8,135.5,134.7\left(\mathrm{~d}, J_{\mathrm{CF}}=9.0 \mathrm{~Hz}\right), 130.3(\mathrm{~d}$, $\left.J_{\mathrm{CF}}=3.0 \mathrm{~Hz}\right), 129.0,128.8,127.6,127.5,127.1,124.6,124.3(\mathrm{~d}$, $\left.J_{\mathrm{CF}}=3.0 \mathrm{~Hz}\right), 122.8,115.9,115.8,108.9,90.9\left(\mathrm{~d}, J_{\mathrm{CF}}=4.5 \mathrm{~Hz}\right)$, 61.6, 44.6, 29.1, 15.9; ESI HRMS: calcd for $\mathrm{C}_{28} \mathrm{H}_{22} \mathrm{FNO}_{4} \mathrm{Na}^{+}$ 478.1431, found 478.1430.

Compound 10c was obtained as white solid, 68\% yield (46.5 $\mathrm{mg}$ ). The diastereomeric ratio was determined to be $16: 1$ by crude ${ }^{1} \mathrm{H}-\mathrm{NMR}$ analysis. Mp 151-152 ${ }^{\circ} \mathrm{C} ;{ }^{1} \mathrm{H}$ NMR $(600 \mathrm{MHz}$, $\left.\mathrm{CDCl}_{3}\right) \delta(\mathrm{ppm}) 7.33-7.32(\mathrm{~m}, 4 \mathrm{H}), 7.31-7.28(\mathrm{~m}, 3 \mathrm{H}), 6.94(\mathrm{td}, J$ $=7.8,1.2 \mathrm{~Hz}, 1 \mathrm{H}), 6.91(\mathrm{dd}, J=7.2,1.2 \mathrm{~Hz}, 1 \mathrm{H}), 6.86(\mathrm{td}, J=7.2$, $0.6 \mathrm{~Hz}, 1 \mathrm{H}), 6.81-6.76(\mathrm{~m}, 1 \mathrm{H}), 6.28$ (d, $J=7.8 \mathrm{~Hz}, 1 \mathrm{H}), 6.25$ (s, $1 \mathrm{H}), 5.04(\mathrm{~d}, J=15.6 \mathrm{~Hz}, 1 \mathrm{H}), 4.38$ (d, $J=15.6 \mathrm{~Hz}, 1 \mathrm{H}), 2.56(\mathrm{~s}$, 3H), 2.16 (s, 3H); ${ }^{13} \mathrm{C}$ NMR (150 MHz, $\left.\mathrm{CDCl}_{3}\right) \delta$ (ppm) 191.4, 191.0, 177.3, 169.9, $165.8\left(\mathrm{~d}, J_{\mathrm{CF}}=255.0 \mathrm{~Hz}\right), 142.3,135.3,131.4$ $\left(\mathrm{d}, J_{\mathrm{CF}}=3.0 \mathrm{~Hz}\right), 130.2\left(\mathrm{~d}, J_{\mathrm{CF}}=9.0 \mathrm{~Hz}\right), 129.1,128.9,127.7$, 127.5, 126.9, 124.8, 122.9, 118.6, 115.6 (d, $\left.J_{\mathrm{CF}}=22.5 \mathrm{~Hz}\right), 108.9$, 88.7, 62.1, 44.7, 29.1, 15.9; ESI HRMS: calcd for $\mathrm{C}_{28} \mathrm{H}_{22} \mathrm{FNO}_{4} \mathrm{Na}^{+}$ 478.1431, found 478.1429 .

Compound 10d was obtained as yellow solid, $71 \%$ yield (53.9 $\mathrm{mg}$ ). The diastereomeric ratio was determined to be $8: 1$ by crude ${ }^{1} \mathrm{H}-\mathrm{NMR}$ analysis. Mp $196-197{ }^{\circ} \mathrm{C} ;{ }^{1} \mathrm{H}$ NMR $(600 \mathrm{MHz}$, $\left.\mathrm{CDCl}_{3}\right) \delta(\mathrm{ppm}) 7.37-7.34(\mathrm{~m}, 3 \mathrm{H}), 7.33(\mathrm{~d}, J=1.8 \mathrm{~Hz}, 2 \mathrm{H}), 7.28$ $(\mathrm{dt}, J=6.0,1.2 \mathrm{~Hz}, 1 \mathrm{H}), 7.12(\mathrm{~d}, J=8.4 \mathrm{~Hz}, 1 \mathrm{H}), 7.08(\mathrm{dd}, J=8.4$, $1.8 \mathrm{~Hz}, 1 \mathrm{H}), 6.98(\mathrm{dt}, J=9.0,4.8 \mathrm{~Hz}, 1 \mathrm{H}), 6.88(\mathrm{~d}, J=4.2 \mathrm{~Hz}, 2 \mathrm{H})$, $6.31(\mathrm{~d}, J=7.8 \mathrm{~Hz}, 1 \mathrm{H}), 6.18(\mathrm{~s}, 1 \mathrm{H}), 5.12(\mathrm{~d}, J=15.6 \mathrm{~Hz}, 1 \mathrm{H})$, $4.36(\mathrm{~d}, J=15.6 \mathrm{~Hz}, 1 \mathrm{H}), 2.55(\mathrm{~s}, 3 \mathrm{H}), 2.16$ (s, 3H); ${ }^{13} \mathrm{C}$ NMR $(150$ $\left.\mathrm{MHz}, \mathrm{CDCl}_{3}\right) \delta$ (ppm) 191.0, 190.9, 177.1, 169.7, 142.3, 138.2, $135.2,134.4,133.3,130.4,129.5,129.3,128.9,127.8,127.5$, $126.7,126.3$, 124.7, 123.0, 118.5, 109.0, 88.8, 62.0, 44.8, 29.1, 15.9; ESI HRMS: calcd for $\mathrm{C}_{28} \mathrm{H}_{21} \mathrm{Cl}_{2} \mathrm{NO}_{4} \mathrm{Na}^{+} 528.0745$, found 528.0748 .

Compound 10e was obtained as white solid, 75\% yield (58.1 $\mathrm{mg}$ ). The diastereomeric ratio was determined to be $10: 1$ by crude ${ }^{1} \mathrm{H}-\mathrm{NMR}$ analysis. Mp $152-153{ }^{\circ} \mathrm{C} ;{ }^{1} \mathrm{H}$ NMR $(400 \mathrm{MHz}$, $\left.\mathrm{CDCl}_{3}\right) \delta(\mathrm{ppm}) 7.35-7.31(\mathrm{~m}, 4 \mathrm{H}), 7.25(\mathrm{t}, J=4.0 \mathrm{~Hz}, 3 \mathrm{H}), 7.12$ $(\mathrm{d}, J=8.4 \mathrm{~Hz}, 2 \mathrm{H}), 6.96(\mathrm{td}, J=7.6,2.0 \mathrm{~Hz}, 1 \mathrm{H}), 6.90(\mathrm{dd}, J=7.2$, $1.4 \mathrm{~Hz}, 1 \mathrm{H}), 6.86(\mathrm{t}, J=7.6 \mathrm{~Hz}, 1 \mathrm{H}), 6.30(\mathrm{~d}, J=8.0 \mathrm{~Hz}, 1 \mathrm{H}), 6.23$ (s, 1H), 5.00 (d, $J=15.6 \mathrm{~Hz}, 1 \mathrm{H}), 4.41$ (d, $J=15.6 \mathrm{~Hz}, 1 \mathrm{H}), 2.55$ (s, 
3H), 2.15 (s, 3H); ${ }^{13} \mathrm{C}$ NMR (100 MHz, $\left.\mathrm{CDCl}_{3}\right) \delta$ (ppm) 191.9, $190.9,177.2$, 169.7, 142.3, 135.2, 133.6, 131.6, 129.1, 128.9, $128.8,128.7,127.7,127.5,127.4$, 126.8, 124.7, 122.8, 118.5, 108.9, 88.7, 61.9, 44.8, 29.0, 15.8; ESI HRMS: calcd for $\mathrm{C}_{28} \mathrm{H}_{22}$ $\mathrm{BrNO}_{4} \mathrm{Na}^{+}$538.0630, found 538.0632.

Compound 10f was obtained as white solid, $78 \%$ yield $(52.8$ $\mathrm{mg}$ ). The diastereomeric ratio was determined to be $20: 1$ by crude ${ }^{1} \mathrm{H}-\mathrm{NMR}$ analysis. Mp $178-179{ }^{\circ} \mathrm{C} ;{ }^{1} \mathrm{H}$ NMR $(600 \mathrm{MHz})$ $\delta(\mathrm{ppm}) 7.31(\mathrm{td}, J=7.8,1.2 \mathrm{~Hz}, 1 \mathrm{H}), 7.28-7.26(\mathrm{~m}, 3 \mathrm{H}), 7.22(\mathrm{t}, J=$ $6.0 \mathrm{~Hz}, 3 \mathrm{H}), 7.12(\mathrm{t}, J=7.2 \mathrm{~Hz}, 1 \mathrm{H}), 7.01(\mathrm{dd}, J=7.2,1.2 \mathrm{~Hz}, 1 \mathrm{H})$, $6.97(\mathrm{td}, J=7.2,2.4 \mathrm{~Hz}, 2 \mathrm{H}), 6.89(\mathrm{td}, J=7.8,0.6 \mathrm{~Hz}, 1 \mathrm{H}), 6.30(\mathrm{~s}$, $1 \mathrm{H}), 6.20(\mathrm{~d}, J=7.8 \mathrm{~Hz}, 1 \mathrm{H}), 4.97(\mathrm{~d}, J=16.2 \mathrm{~Hz}, 1 \mathrm{H}), 4.02(\mathrm{~d}, J=$ $16.2 \mathrm{~Hz}, 1 \mathrm{H}), 2.56$ (s, 3H), 2.13 (s, 3H), 1.81 (s, 3H); ${ }^{13} \mathrm{C}$ NMR (150 $\left.\mathrm{MHz}, \mathrm{CDCl}_{3}\right) \delta(\mathrm{ppm}) 194.3,190.9,176.9,169.6,142.3,140.1$, 140.0, 135.2, 133.9, 132.3, 131.7, 129.1, 128.7, 128.5, 127.4, 127.0, 125.1, 124.5, 122.8, 118.9, 109.1, 89.4, 61.8, 44.5, 28.9, 20.0, 15.8; ESI HRMS: calcd for $\mathrm{C}_{29} \mathrm{H}_{25} \mathrm{NO}_{4} \mathrm{Na}^{+} 474.1681$, found 474.1683 .

Compound $10 \mathrm{~g}$ was obtained as white solid, $81 \%$ yield (56.8 $\mathrm{mg})$. The diastereomeric ratio was determined to be $18: 1$ by crude ${ }^{1} \mathrm{H}-\mathrm{NMR}$ analysis. Mp $188-189{ }^{\circ} \mathrm{C} ;{ }^{1} \mathrm{H}$ NMR $(600 \mathrm{MHz}$, $\left.\mathrm{CDCl}_{3}\right) \delta(\mathrm{ppm}) 7.33(\mathrm{t}, J=3.0 \mathrm{~Hz}, 1 \mathrm{H}), 7.31(\mathrm{t}, J=1.8 \mathrm{~Hz}, 1 \mathrm{H})$, 7.29-7.28 (m, 4H), 7.26-7.24 (m, 1H), $6.94(\mathrm{~d}, J=7.2 \mathrm{~Hz}, 1 \mathrm{H}), 6.92$ $(\mathrm{dd}, J=7.2,1.2 \mathrm{~Hz}, 1 \mathrm{H}), 6.85(\mathrm{td}, J=7.2,0.6 \mathrm{~Hz}, 1 \mathrm{H}), 6.63(\mathrm{dt}, J=$ 9.6, $3.0 \mathrm{~Hz}, 2 \mathrm{H}), 6.26(\mathrm{~d}, J=7.2 \mathrm{~Hz}, 2 \mathrm{H}), 5.03(\mathrm{~d}, J=15.6 \mathrm{~Hz}, 1 \mathrm{H})$, $4.46(\mathrm{~d}, J=16.2 \mathrm{~Hz}, 1 \mathrm{H}), 3.78(\mathrm{~s}, 3 \mathrm{H}), 2.55(\mathrm{~s}, 3 \mathrm{H}), 2.15(\mathrm{~s}, 3 \mathrm{H}) ;{ }^{13} \mathrm{C}$ NMR (150 MHz, $\mathrm{CDCl}_{3}$ ) $\delta$ (ppm) 191.1, 191.0, 177.5, 170.1, 163.9, 142.3, 135.5, 130.0, 128.9, 128.8, 128.0, 127.5, 127.4, 127.0, 124.9, 122.8, 118.6, 113.7, 108.8, 88.5, 62.2, 55.6, 44.7, 29.1, 16.0; ESI HRMS: calcd for $\mathrm{C}_{29} \mathrm{H}_{25} \mathrm{NO}_{5} \mathrm{Na}^{+} 490.1630$, found 490.1631 .

Compound 10h was obtained as white solid, $60 \%$ yield (39.9 $\mathrm{mg}$ ). The diastereomeric ratio was determined to be $5: 1$ by crude ${ }^{1} \mathrm{H}-\mathrm{NMR}$ analysis. Mp $172-173{ }^{\circ} \mathrm{C} ;{ }^{1} \mathrm{H}$ NMR $(600 \mathrm{MHz}$, $\left.\mathrm{CDCl}_{3}\right) \delta(\mathrm{ppm}) 7.51(\mathrm{dd}, J=4.8,1.2 \mathrm{~Hz}, 1 \mathrm{H}), 7.36(\mathrm{dd}, J=4.2$, $1.2 \mathrm{~Hz}, 3 \mathrm{H}), 7.34-7.31(\mathrm{~m}, 2 \mathrm{H}), 7.28(\mathrm{dd}, J=6.6,1.2 \mathrm{~Hz}, 1 \mathrm{H}), 6.98$ $(\mathrm{td}, J=7.8,1.2 \mathrm{~Hz}, 1 \mathrm{H}), 6.95(\mathrm{dd}, J=7.2,0.6 \mathrm{~Hz}, 1 \mathrm{H}), 6.88(\mathrm{dd}, J$ $=7.2,0.6 \mathrm{~Hz}, 1 \mathrm{H}), 6.83-6.82(\mathrm{~m}, 1 \mathrm{H}), 6.40(\mathrm{~d}, J=7.8 \mathrm{~Hz}, 1 \mathrm{H})$, $6.08(\mathrm{~s}, 1 \mathrm{H}), 5.06(\mathrm{~d}, J=15.6 \mathrm{~Hz}, 1 \mathrm{H}), 4.64(\mathrm{~d}, J=15.6 \mathrm{~Hz}, 1 \mathrm{H})$, $2.56(\mathrm{~s}, 3 \mathrm{H}), 2.16(\mathrm{~s}, 3 \mathrm{H}) ;{ }^{13} \mathrm{C} \mathrm{NMR}\left(150 \mathrm{MHz}, \mathrm{CDCl}_{3}\right) \delta(\mathrm{ppm})$ $191.2,184.9,177.5,169.9,142.4,140.9,135.5,135.4,132.2$, $129.1,128.9,128.8,127.9,127.7,127.5,124.8$, 123.0, 118.6, 108.9, 89.2, 62.6, 44.7, 29.1, 15.9; ESI HRMS: calcd for $\mathrm{C}_{26} \mathrm{H}_{21}$ $\mathrm{NO}_{4} \mathrm{SNa}^{+} 466.1089$, found 466.1090 .

Compound 10i was obtained as white solid, $62 \%$ yield $(45.3$ $\mathrm{mg}$ ). The diastereomeric ratio was determined to be $5: 1$ by crude ${ }^{1} \mathrm{H}$-NMR analysis. Mp $170-171{ }^{\circ} \mathrm{C} ;{ }^{1} \mathrm{H}$ NMR $(400 \mathrm{MHz}$, $\left.\mathrm{CDCl}_{3}\right) \delta(\mathrm{ppm}) 7.90-7.86(\mathrm{~m}, 2 \mathrm{H}), 7.80(\mathrm{~d}, J=8.0 \mathrm{~Hz}, 1 \mathrm{H}), 7.63-$ $7.53(\mathrm{~m}, 3 \mathrm{H}), 7.27$ (d, $J=7.2 \mathrm{~Hz}, 2 \mathrm{H}), 7.09-7.08(\mathrm{~m}, 4 \mathrm{H}), 6.97$ (d, $=7.2 \mathrm{~Hz}, 1 \mathrm{H}), 6.87-6.83(\mathrm{~m}, 2 \mathrm{H}), 6.48(\mathrm{~s}, 1 \mathrm{H}), 5.95(\mathrm{~d}, J=7.2 \mathrm{~Hz}$, $1 \mathrm{H}), 5.00$ (d, $J=16.0 \mathrm{~Hz}, 1 \mathrm{H}), 3.76(\mathrm{~d}, J=16.0 \mathrm{~Hz}, 1 \mathrm{H}), 2.59$ (s, 3H), $2.16(\mathrm{~s}, 3 \mathrm{H}) ;{ }^{13} \mathrm{C}$ NMR (150 MHz, $\left.\mathrm{CDCl}_{3}\right) \delta(\mathrm{ppm})$ 192.7, 190.9 , 177.3, 169.9, 142.0, 135.4, 134.9, 132.3, 131.8, 129.5, $129.1,128.9,128.8,128.5,128.3,127.7,127.3$, 127.0, 126.9, 124.7, 123.0, 122.7, 118.4, 108.8, 89.0, 62.1, 44.4, 29.7, 29.0, 15.9; ESI HRMS: calcd for $\mathrm{C}_{32} \mathrm{H}_{25} \mathrm{NO}_{4} \mathrm{Na}^{+} 510.1681$, found 510.1682 .

Compound $10 \mathrm{j}$ was obtained as white solid, $74 \%$ yield $(41.7$ $\mathrm{mg}$ ). The diastereomeric ratio was determined to be $6: 1$ by crude ${ }^{1} \mathrm{H}-\mathrm{NMR}$ analysis. Mp $145-146{ }^{\circ} \mathrm{C} ;{ }^{1} \mathrm{H}$ NMR $(600 \mathrm{MHz}$,
$\left.\mathrm{CDCl}_{3}\right) \delta(\mathrm{ppm}) 7.48(\mathrm{~d}, J=7.2 \mathrm{~Hz}, 2 \mathrm{H}), 7.36(\mathrm{t}, J=7.8 \mathrm{~Hz}, 3 \mathrm{H})$, $7.29(\mathrm{~d}, J=7.2 \mathrm{~Hz}, 1 \mathrm{H}), 7.15(\mathrm{dt}, J=3.0,1.2 \mathrm{~Hz}, 1 \mathrm{H}), 6.93(\mathrm{~d}, J=$ $2.4 \mathrm{~Hz}, 1 \mathrm{H}), 6.71(\mathrm{~d}, J=7.8 \mathrm{~Hz}, 1 \mathrm{H}), 5.38(\mathrm{~s}, 1 \mathrm{H}), 5.10$ (d, $J=$ $16.2 \mathrm{~Hz}, 1 \mathrm{H}), 4.99$ (d, $J=15.6 \mathrm{~Hz}, 1 \mathrm{H}), 2.50(\mathrm{~s}, 3 \mathrm{H}), 2.12(\mathrm{~s}, 3 \mathrm{H})$, 1.77 (s, 3H); ${ }^{13} \mathrm{C}$ NMR (150 MHz, $\left.\mathrm{CDCl}_{3}\right) \delta$ (ppm) 201.4, 191.1, $176.9,169.3,142.6,135.5,129.4,128.8,127.7,127.4,123.5$, 122.9, 109.6, 91.7, 61.2, 44.7, 28.9, 27.3, 15.7; ESI HRMS: calcd for $\mathrm{C}_{23} \mathrm{H}_{21} \mathrm{NO}_{4} \mathrm{Na}^{+}$398.1368, found 398.1368.

Compound 10k was obtained as white solid, $76 \%$ yield (46.2 $\mathrm{mg}$ ). The diastereomeric ratio was determined to be $5: 1$ by crude ${ }^{1} \mathrm{H}-\mathrm{NMR}$ analysis. Mp $168-169{ }^{\circ} \mathrm{C} ;{ }^{1} \mathrm{H}$ NMR $(600 \mathrm{MHz}$, $\left.\mathrm{CDCl}_{3}\right) \delta(\mathrm{ppm}) 7.44(\mathrm{~d}, J=7.8 \mathrm{~Hz}, 2 \mathrm{H}), 7.34(\mathrm{t}, J=7.2 \mathrm{~Hz}, 2 \mathrm{H})$, $7.28(\mathrm{~d}, J=7.2 \mathrm{~Hz}, 1 \mathrm{H}), 7.14(\mathrm{td}, J=7.8,1.2 \mathrm{~Hz}, 1 \mathrm{H}), 7.02(\mathrm{dd}, J=$ $7.8,1.2 \mathrm{~Hz}, 1 \mathrm{H}), 6.93(\mathrm{td}, J=7.8,0.6 \mathrm{~Hz}, 1 \mathrm{H}), 6.67(\mathrm{~d}, J=7.8 \mathrm{~Hz}$, $1 \mathrm{H}), 5.43(\mathrm{~s}, 1 \mathrm{H}), 5.17$ (d, $J=15.6 \mathrm{~Hz}, 1 \mathrm{H}), 4.86$ (d, $J=15.6 \mathrm{~Hz}$, $1 \mathrm{H}), 4.20(\mathrm{~m}, 1 \mathrm{H}), 3.72(\mathrm{~m}, 1 \mathrm{H}), 2.50(\mathrm{~s}, 3 \mathrm{H}), 2.13(\mathrm{~s}, 3 \mathrm{H}), 0.50(\mathrm{t}$, $J=7.2 \mathrm{~Hz}, 3 \mathrm{H}) ;{ }^{13} \mathrm{C} \mathrm{NMR}\left(150 \mathrm{MHz}, \mathrm{CDCl}_{3}\right) \delta(\mathrm{ppm}) 191.2,176.9$, 169.5 , 166.1, 143.0, 135.8, 128.3, 128.8, 128.0, 127.7, 123.7, 122.7, 109.2, 85.7, 61.5, 44.6, 34.4, 31.8, 29.0, 15.8, 13.3; ESI HRMS: calcd for $\mathrm{C}_{24} \mathrm{H}_{23} \mathrm{NO}_{5} \mathrm{Na}^{+} 428.1474$, found 428.1476 .

Compound 101 was obtained as white solid, $70 \%$ yield ( 47.8 $\mathrm{mg}$ ). The diastereomeric ratio was determined to be $10: 1$ by crude ${ }^{1} \mathrm{H}$-NMR analysis. Mp 158-159 ${ }^{\circ} \mathrm{C} ;{ }^{1} \mathrm{H}$ NMR $(600 \mathrm{MHz}$, $\left.\mathrm{CDCl}_{3}\right) \delta(\mathrm{ppm}) 7.46(\mathrm{tt}, J=7.8,1.2 \mathrm{~Hz}, 1 \mathrm{H}), 7.33(\mathrm{dd}, J=8.4$, $1.2 \mathrm{~Hz}, 2 \mathrm{H}), 7.31-7.28(\mathrm{~m}, 4 \mathrm{H}), 7.20(\mathrm{td}, J=7.8,1.8 \mathrm{~Hz}, 2 \mathrm{H}), 6.69$ $(\mathrm{dd}, J=7.8,2.4 \mathrm{~Hz}, 1 \mathrm{H}), 6.60(\mathrm{td}, J=8.4,2.4 \mathrm{~Hz}, 1 \mathrm{H}), 6.31(\mathrm{~s}$, $1 \mathrm{H}), 6.07(\mathrm{dd}, J=8.4,4.2 \mathrm{~Hz}, 1 \mathrm{H}), 5.30(\mathrm{~s}, 1 \mathrm{H}), 5.10(\mathrm{~d}, J=$ $16.0 \mathrm{~Hz}, 1 \mathrm{H}), 4.18$ (d, J=16.2 Hz, 1H), 2.57 (s, 3H), 2.22 (s, 3H); ${ }^{13} \mathrm{C} \mathrm{NMR}\left(150 \mathrm{MHz}, \mathrm{CDCl}_{3}\right) \delta(\mathrm{ppm})$ 192.6, 190.9, 177.2, 159.2 (d, $\left.J_{\mathrm{CF}}=241.5 \mathrm{~Hz}\right), 138.3,135.0,134.9,128.9,128.5,127.7,127.6$, 127.2, 118.6, $115.3\left(\mathrm{~d}, J_{\mathrm{CF}}=24.0 \mathrm{~Hz}\right), 112.7\left(\mathrm{~d}, J_{\mathrm{CF}}=24.0 \mathrm{~Hz}\right)$, $109.4\left(\mathrm{~d}, J_{\mathrm{CF}}=9.0 \mathrm{~Hz}\right), 88.8,62.4$, 44.8, 29.1, 15.9; ESI HRMS: calcd for $\mathrm{C}_{28} \mathrm{H}_{22} \mathrm{FNO}_{4} \mathrm{Na}^{+}$478.1431, found 478.1429 .

Compound $10 \mathrm{~m}$ was obtained as white solid, $56 \%$ yield $(38.2$ $\mathrm{mg}$ ). The diastereomeric ratio was determined to be $16: 1$ by crude ${ }^{1} \mathrm{H}-\mathrm{NMR}$ analysis. Mp 160-161 ${ }^{\circ} \mathrm{C} ;{ }^{1} \mathrm{H}$ NMR $(600 \mathrm{MHz}$, $\left.\mathrm{CDCl}_{3}\right) \delta(\mathrm{ppm}) 7.43(\mathrm{tt}, J=7.8,1.2 \mathrm{~Hz}, 1 \mathrm{H}), 7.39(\mathrm{~d}, J=7.2 \mathrm{~Hz}$, $2 \mathrm{H}), 7.32(\mathrm{td}, J=7.2,1.8 \mathrm{~Hz}, 2 \mathrm{H}), 7.28-7.25(\mathrm{~m}, 1 \mathrm{H}), 7.23(\mathrm{dd}, J=$ 8.4, 1.2 Hz, 2H), $7.12(\mathrm{t}, J=7.2 \mathrm{~Hz}, 2 \mathrm{H}), 6.82-6.79(\mathrm{~m}, 1 \mathrm{H}), 6.72-$ $6.68(\mathrm{~m}, 2 \mathrm{H}), 6.27(\mathrm{~s}, 1 \mathrm{H}), 5.09(\mathrm{~d}, J=15.6 \mathrm{~Hz}, 1 \mathrm{H}), 4.48(\mathrm{~d}, J=$ $15.0 \mathrm{~Hz}, 1 \mathrm{H}), 2.56(\mathrm{~s}, 3 \mathrm{H}), 2.16(\mathrm{~s}, 3 \mathrm{H}) ;{ }^{13} \mathrm{C} \mathrm{NMR}\left(150 \mathrm{MHz}, \mathrm{CDCl}_{3}\right)$ $\delta(\mathrm{ppm}) 192.7,191.0,177.1,170.1,146.9\left(\mathrm{~d}, J_{\mathrm{CF}}=244.9 \mathrm{~Hz}\right), 136.6$, 134.8, 133.8, 128.6, 128.4, 127.6 (d, $\left.J_{\mathrm{CF}}=2.0 \mathrm{~Hz}\right), 127.4,123.5$, 123.4, 120.7, 120.6, 118.6, 117.2, 117.0, 89.0, 62.2, 46.1, 29.1, 15.9; ESI HRMS: calcd for $\mathrm{C}_{28} \mathrm{H}_{22} \mathrm{FNO}_{4} \mathrm{Na}^{+} 478.1431$, found 478.1432 .

Compound 10n was obtained as white solid, 66\% yield (46.7 $\mathrm{mg}$ ). The diastereomeric ratio was determined to be $5: 1$ by crude ${ }^{1} \mathrm{H}-\mathrm{NMR}$ analysis. Mp 159-160 ${ }^{\circ} \mathrm{C} ;{ }^{1} \mathrm{H}$ NMR (600 $\mathrm{MHz}, \mathrm{CDCl}_{3}$ ) $\delta(\mathrm{ppm}) 7.46(\mathrm{t}, J=7.8 \mathrm{~Hz}, 1 \mathrm{H}), 7.31(\mathrm{dd}, J=7.8,1.2 \mathrm{~Hz}, 3 \mathrm{H}), 7.28$ $(\mathrm{d}, J=5.4 \mathrm{~Hz}, 3 \mathrm{H}), 7.24(\mathrm{dd}, J=6.6,5.4 \mathrm{~Hz}, 1 \mathrm{H}), 7.21(\mathrm{t}, J=7.8 \mathrm{~Hz}$, $2 \mathrm{H}), 6.91(\mathrm{~d}, J=1.8 \mathrm{~Hz}, 1 \mathrm{H}), 6.87(\mathrm{dd}, J=8.4,2.4 \mathrm{~Hz}, 1 \mathrm{H}), 6.29(\mathrm{~s}$, $1 \mathrm{H}), 6.06(\mathrm{~d}, J=8.4 \mathrm{~Hz}, 1 \mathrm{H}), 5.08(\mathrm{~d}, J=16.2 \mathrm{~Hz}, 1 \mathrm{H}), 4.18(\mathrm{~d}, J=$ $16.2 \mathrm{~Hz}, 1 \mathrm{H}), 2.57$ (s, 3H), $2.24(\mathrm{~s}, 3 \mathrm{H}) ;{ }^{13} \mathrm{C} \mathrm{NMR}\left(150 \mathrm{MHz}, \mathrm{CDCl}_{3}\right)$ $\delta(\mathrm{ppm}) 192.7,191.0,177.1,170.1,147.7,146.1,136.6,134.8$, 133.8, 128.6, 128.4, 127.7, 127.6, 127.5, 127.4, 123.5, 123.4, 120.6, 118.6, 117.2, 117.0, 89.0, 62.2, 46.1, 29.0, 15.9; ESI HRMS: calcd for $\mathrm{C}_{28} \mathrm{H}_{22} \mathrm{ClNO}_{4} \mathrm{Na}^{+}$494.1135, found 494.1133. 
Compound 100 was obtained as yellow solid, $67 \%$ yield (47.4 $\mathrm{mg}$ ). The diastereomeric ratio was determined to be $15: 1$ by crude ${ }^{1} \mathrm{H}-\mathrm{NMR}$ analysis. Mp $164-165{ }^{\circ} \mathrm{C} ;{ }^{1} \mathrm{H}$ NMR $(600 \mathrm{MHz}$, $\left.\mathrm{CDCl}_{3}\right) \delta(\mathrm{ppm}) 7.47(\mathrm{tt}, J=7.2,1.2 \mathrm{~Hz}, 1 \mathrm{H}), 7.33-7.31(\mathrm{~m}, 1 \mathrm{H})$, $7.31-7.28(\mathrm{~m}, 5 \mathrm{H}), 7.21(\mathrm{td}, J=8.4,1.8 \mathrm{~Hz}, 2 \mathrm{H}), 6.84(\mathrm{~d}, J=$ $1.8 \mathrm{~Hz}, 2 \mathrm{H}), 6.28(\mathrm{~s}, 1 \mathrm{H}), 6.16(\mathrm{~s}, 1 \mathrm{H}), 5.05(\mathrm{~d}, J=15.6 \mathrm{~Hz}, 1 \mathrm{H})$, $4.18(\mathrm{~d}, J=16.2 \mathrm{~Hz}, 1 \mathrm{H}), 2.56(\mathrm{~s}, 3 \mathrm{H}), 2.20(\mathrm{~s}, 3 \mathrm{H}) ;{ }^{13} \mathrm{C}$ NMR $(100$ $\left.\mathrm{MHz}, \mathrm{CDCl}_{3}\right) \delta$ (ppm) 192.7, 190.9, 177.4, 170.0, 143.5, 134.9, $134.7,133.8$, 129.0, 128.5, 128.9, 127.8, 127.5, 125.6, 125.5, 122.7, 118.6, 109.4, 88.8, 61.8, 44.7, 29.1, 15.9; ESI HRMS: calcd for $\mathrm{C}_{28} \mathrm{H}_{22} \mathrm{ClNO}_{4} \mathrm{Na}^{+}$494.1135, found 494.1136.

Compound 10p was obtained as white solid, 70\% yield (54.2 $\mathrm{mg}$ ). The diastereomeric ratio was determined to be $13: 1$ by crude ${ }^{1} \mathrm{H}-\mathrm{NMR}$ analysis. Mp 200-201 ${ }^{\circ} \mathrm{C} ;{ }^{1} \mathrm{H}$ NMR $(600 \mathrm{MHz}$, $\left.\mathrm{CDCl}_{3}\right) \delta(\mathrm{ppm}) 7.46(\mathrm{td}, J=7.2,0.6 \mathrm{~Hz}, 1 \mathrm{H}), 7.31(\mathrm{~d}, J=7.8 \mathrm{~Hz}$, $3 \mathrm{H}), 7.29-7.27(\mathrm{~m}, 4 \mathrm{H}), 7.21(\mathrm{t}, J=7.2 \mathrm{~Hz}, 2 \mathrm{H}), 7.04(\mathrm{~d}, J=$ $1.8 \mathrm{~Hz}, 1 \mathrm{H}), 7.02(\mathrm{dt}, J=8.4,2.4 \mathrm{~Hz}, 1 \mathrm{H}), 6.29(\mathrm{~s}, 1 \mathrm{H}), 6.02(\mathrm{dd}, J$ $=7.8,0.6 \mathrm{~Hz}, 1 \mathrm{H}), 5.08(\mathrm{~d}, J=15.6 \mathrm{~Hz}, 1 \mathrm{H}), 4.18(\mathrm{~d}, J=16.2 \mathrm{~Hz}$, $1 \mathrm{H}), 2.57(\mathrm{~s}, 3 \mathrm{H}), 2.24(\mathrm{~s}, 3 \mathrm{H}) ;{ }^{13} \mathrm{C} \mathrm{NMR}\left(150 \mathrm{MHz}, \mathrm{CDCl}_{3}\right)$ $\delta(\mathrm{ppm}) 192.6,190.9,176.9,170.1,141.4,135.0,134.8,133.7$, $131.8,128.2$, 128.9, 128.5, 127.7, 127.7, 127.6, 127.2, 118.6, 115.4, 110.3, 88.8, 62.0, 44.7, 29.1, 16.0; ESI HRMS: calcd for $\mathrm{C}_{28} \mathrm{H}_{22} \mathrm{BrNO}_{4} \mathrm{Na}^{+}$538.0630, found 538.0632.

Compound 10q was obtained as yellow solid, 69\% yield (54.5 $\mathrm{mg}$ ). The diastereomeric ratio was determined to be $10: 1$ by crude ${ }^{1} \mathrm{H}-\mathrm{NMR}$ analysis. Mp 184-185 ${ }^{\circ} \mathrm{C} ;{ }^{1} \mathrm{H}$ NMR (600 $\mathrm{MHz}, \mathrm{CDCl}_{3}$ ) $\delta(\mathrm{ppm}) 7.47(\mathrm{tt}, J=7.8,1.2 \mathrm{~Hz}, 1 \mathrm{H}), 7.32-7.31$ (m, 2H), 7.30-7.26 $(\mathrm{m}, 5 \mathrm{H}), 7.21(\mathrm{td}, J=7.2,1.2 \mathrm{~Hz}, 2 \mathrm{H}), 7.00(\mathrm{dd}, J=7.8,1.8 \mathrm{~Hz}, 1 \mathrm{H})$, $6.78(\mathrm{~d}, J=7.8 \mathrm{~Hz}, 1 \mathrm{H}), 6.31(\mathrm{~d}, J=1.8 \mathrm{~Hz}, 1 \mathrm{H}), 6.28(\mathrm{~s}, 1 \mathrm{H}), 5.05(\mathrm{~d}$, $J=15.6 \mathrm{~Hz}, 1 \mathrm{H}), 4.18(\mathrm{~d}, J=16.2 \mathrm{~Hz}, 1 \mathrm{H}), 2.55$ (s, 3H), 2.21 (s, 3H); ${ }^{13} \mathrm{C}$ NMR (150 MHz, $\left.\mathrm{CDCl}_{3}\right) \delta(\mathrm{ppm})$ 192.7, 190.9, 177.3, 170.0, 143.6, 134.8, 134.7, 133.8, 128.9, 128.5, 127.8, 127.5, 127.2, 126.1, 125.9, 125.7, 122.6, 118.5, 112.1, 88.7, 61.8, 44.7, 29.1, 15.9; ESI HRMS: calcd for $\mathrm{C}_{28} \mathrm{H}_{22} \mathrm{BrNO}_{4} \mathrm{Na}^{+} 538.0630$, found 538.0633.

Compound 10r was obtained as white solid, $80 \%$ yield $(54.1$ $\mathrm{mg}$ ). The diastereomeric ratio was determined to be $18: 1$ by crude ${ }^{1} \mathrm{H}-\mathrm{NMR}$ analysis. Mp $162-163{ }^{\circ} \mathrm{C} ;{ }^{1} \mathrm{H}$ NMR $(400 \mathrm{MHz}$, $\left.\mathrm{CDCl}_{3}\right) \delta(\mathrm{ppm}) 7.42(\mathrm{t}, J=7.2 \mathrm{~Hz}, 1 \mathrm{H}), 7.25(\mathrm{~d}, J=4.4 \mathrm{~Hz}, 4 \mathrm{H})$, $7.25(\mathrm{~d}, J=7.2 \mathrm{~Hz}, 3 \mathrm{H}), 7.16(\mathrm{t}, J=7.6 \mathrm{~Hz}, 2 \mathrm{H}), 6.74(\mathrm{~s}, 1 \mathrm{H}), 6.70$ $(\mathrm{d}, J=8.0 \mathrm{~Hz}, 1 \mathrm{H}), 6.29(\mathrm{~s}, 1 \mathrm{H}), 6.05(\mathrm{~d}, J=8.0 \mathrm{~Hz}, 1 \mathrm{H}), 5.06(\mathrm{~d}, J$ $=16.0 \mathrm{~Hz}, 1 \mathrm{H}), 4.16(\mathrm{~d}, J=16.0 \mathrm{~Hz}, 1 \mathrm{H}), 2.56(\mathrm{~s}, 3 \mathrm{H}), 2.18(\mathrm{~s}$, $3 \mathrm{H}), 2.13$ (s, 3H); ${ }^{13} \mathrm{C} \mathrm{NMR}\left(100 \mathrm{MHz}, \mathrm{CDCl}_{3}\right) \delta$ (ppm) 193.0, $191.1,177.1,169.9,139.8,135.4,135.1,133.3,132.3,129.2$, $128.7,128.5,128.4,128.3,127.5$, 127.4, 127.3, 126.9, 125.4, 118.4 108.5, 89.1, 62.0, 44.6, 29.0, 20.9, 15.9; ESI HRMS: calcd for $\mathrm{C}_{29} \mathrm{H}_{25} \mathrm{NO}_{4} \mathrm{Na}^{+}$474.1681, found 474.1683.

\section{Conflicts of interest}

There are no conflicts to declare.

\section{Acknowledgements}

We are grateful for financial support from the National Natural Science Foundation of China (81573588, 81773889, 21702035), the Science \& Technology Department of Sichuan Province (2017JZYD0001, 2017JY0323, 2016TD0006).

\section{Notes and references}

1 For selective reviews on spirooxindole, see:(a) B. Yu, Y.-C. Zheng, X.-J. Shi, P.-P. Qi and H.-M. Liu, Anti-Cancer Agents Med. Chem., 2016, 16, 1315-1324; (b) M. Somei and F. Yamada, Nat. Prod. Rep., 2005, 22, 73-103; (c) A. J. Kochanowska-Karamyan and M. T. Hamann, Chem. Rev., 2010, 110, 4489-4497; (d) J. J. Badillo, N. V. Hanhan and A. K. Franz, Curr. Opin. Drug Discovery Dev., 2010, 13, 758-776; (e) C. V. Galliford and K. A. Scheidt, Angew. Chem., Int. Ed., 2007, 46, 8748-8758.

2 (a) Z. Zhang, Z. Wang, K. Huang, Y. Liu, C. Wei, J. Zhou, W. Zhang, Q. Wang, H. Liang, A. Zhang, G. Wang, Y. Zhen and L. Han, Cancer Lett., 2019, 443, 91-107; (b) C.-B. Cui, H. Kakeya and H. Osada, Tetrahedron, 1996, 52, 1265112666; (c) B. Yu, D.-Q. Yu and H.-M. Liu, Eur. J. Med. Chem., 2015, 97, 673-698.

3 For selective reviews on the synthesis of spirooxindole, see:(a) G. S. Singh and Z. Y. Desta, Chem. Rev., 2012, 112, 6104-6155; (b) D. Cheng, Y. Ishihara, B. Tan and C. F. Barbas, III, ACS Catal., 2014, 4, 743-762; (c) G.-J. Mei and F. Shi, Chem. Commun., 2018, 54, 6607-6621; (d) M. Xia and R.-Z. Ma, J. Heterocycl. Chem., 2014, 51, 539554; (e) M. M. M. Santos, Tetrahedron, 2014, 70, 9735-9757; (f) Y. Liu, H. Wang and J. Wan, Asian J. Org. Chem., 2013, 2, 374-386; (g) A. K. Franz, N. V. Hanhan and N. R. BallJones, ACS Catal., 2013, 3, 540-553; (h) L. Hong and R. Wang, Adv. Synth. Catal., 2013, 355, 1023-1052; (i) N. R. Ball-Jones, J. J. Badillo and A. K. Franz, Org. Biomol. Chem., 2012, 10, 5165-5181; (j) J. P. MacDonald, J. J. Badillo, G. E. Arevalo, A. Silva-García and A. K. Franz, ACS Comb. Sci., 2012, 14, 285-293. For the lasted examples on the synthesis of spirooxindole, see: $(k)$ C.-S. Wang, T.-Z. Li, Y.-C. Cheng, J. Zhou, G.-J. Mei and F. Shi, J. Org. Chem., 2019, 84, 3214-3222; (l) F. Jiang, G.-Z. Luo, Z.-Q. Zhu, C.-S. Wang, G.-J. Mei and F. Shi, J. Org. Chem., 2018, 83, 10060-10069; ( $m$ ) X.-L. Jiang, S.-J. Liu, Y.-Q. Gu, G.-J. Mei and F. Shi, Adv. Synth. Catal., 2017, 359, 33413346; (n) X.-L. Lian, A. Adili, B. Liu, Z.-L. Tao and Z.-Y. Han, Org. Biomol. Chem., 2017, 15, 3670-3673; (o) H. J. Roh, S. Y. Kim, B. K. Min and J. N. kim, Tetrahedron Lett., 2017, 58, 21-24; (p) G. Zhan, M.-L. Shi, Q. He, W.-J. Lin, Q. Ouyang, W. Du and Y.-C. Chen, Angew. Chem., Int. Ed., 2016, 55, 2147-2151; (q) W.-L. Chan, X. Tang, F. Zhang, G. Quek, G.-J. Mei and Y. Lu, Angew. Chem., Int. Ed., 2019, DOI: 10.1002/anie.201900758.

4 (a) A. Kimishima, H. Umihara, A. Mizoguchi, S. Yokoshima and T. Fukuyama, Org. Lett., 2014, 16, 6244-6247; (b) B. W. Son, P. R. Jensen, C. A. Kaulfman and W. Fenical, Nat. Prod. Rep., 1999, 13, 213-222; (c) P. M. A. Calverley, J. A. Anderson, B. Celli, G. T. Ferguson, C. Jenkins, P. W. Jones, J. C. Yates and J. Vestbo, N. Engl. J. Med., 2007, 356, 775-789.

5 (a) Y. Liu, S.-J. Han, W.-B. Liu and B. M. Stoltz, Acc. Chem. Res., 2015, 48, 740-751; (b) R. Long, J. Huang, J. Gong and Z. Yang, Nat. Prod. Rep., 2015, 32, 1584-1601; (c) 
E. A. Peterson and L. E. Overman, Proc. Natl. Acad. Sci. U. S. A., 2004, 101, 11943-11948.

6 For selective books about sulfur ylide, see:(a) V. K. Aggarwal and J. Richardson, in Science of Synthesis : Sulfur Ylides, ed. A.Padwa and D.Bellus, George Thieme Verlag, Stuttgart, 2004, pp. 21-105; (b) V. K. Aggarwal, J. Richardson and C. L. Winn, in Science of Synthesis: $\alpha$-Substituted Sulfur Ylides, ed. A. B.Charette, George Thieme Verlag, Stuttgart, 2005, pp. 11-74, For selective reviews on sulur ylide, see: ; (c) L.-Q. Lu, T.-R. Li, Q. Wang and W.-J. Xiao, Chem. Soc. Rev., 2017, 46, 4135-4149; (d) C. Zhu, Y. Ding and L.-W. Ye, Org. Biomol. Chem., 2015, 13, 2530-2536; (e) X.-L. Sun and Y. Tang, Acc. Chem. Res., 2008, 41, 937-948; (f) V. K. Aggarwal and C. L. Winn, Acc. Chem. Res., 2004, 37, 611-620; $(g)$ A.-H. Li, L.-X. Dai and V. K. Aggarwal, Chem. Rev., 1997, 97, 2341-2372.

7 (a) L. Wang, W. Cao, H. Mei, L. Hu and X. Feng, Adv. Synth. Catal., 2018, 360, 4089-4093; (b) H. Mei, G. Pan, X. Zhang, L. Lin, X. Liu and X. Feng, Org. Lett., 2018, 20, 7794-7797; (c) Q.-Z. Li, X. Zhang, R. Zeng, Q.-S. Dai, Y. Liu, X.-D. Shen, H.-J. Leng, K.-C. Yang and J.-L. Li, Org. Lett., 2018, 20, 3700-3704; (d) Z. Yuan, X. Fang, X. Li, J. Wu, H. Yao and A. Lin, J. Org. Chem., 2015, 80, 11123-11130; (e) X.-Z. Zhang, J.-Y. Du, Y.-H. Deng, W.-D. Chu, X. Yan, K.-Y. Yu and C.-A. Fan, J. Org. Chem., 2016, 81, 2598-2606; (f) L. Liu, Z. Yuan, R. Pan, Y. Zeng, A. Lin, H. Yao and Y. Huang, Org. Chem. Front., 2018, 5, 623-628; $(g)$ S. Ye, Z.-Z. Huang, C.-A. Xia, Y. Tang and L.-X. Dai, J. Am. Chem. Soc., 2002, 124, 2432-2433; (h) X.-M. Deng, P. Cai, S. Ye, X.-L. Sun, W.-W. Liao, K. Li, Y. Tang, Y.-D. Wu and L.-X. Dai, J. Am. Chem. Soc., 2006, 128, 9730-9740; (i) M. A. Marsini, J. T. Reeves, J.-N. Desrosiers, M. A. Herbage, J. Savoie, Z. Li, K. R. Fandrick, C. A. Sader, B. McKibben, D. A. Gao, J. Cui, N. C. Gonnella, H. Lee, X. Wei, F. Roschangar, B. Z. Lu and C. H. Senanayake, Org. Lett., 2015, 17, 5614-5617; (j) H.-Y. Wu, C.-W. Chang and R.-J. Chein, J. Org. Chem., 2013, 78, 5788-5793; (k) O. Illa, M. Namutebi, C. Saha, M. Ostovar, C. C. Chen, M. F. Haddow, S. Nocquet-Thibault, M. Lusi, E. M. McGarrigle and V. K. Aggarwal, J. Am. Chem. Soc., 2013, 135, 11951-11966; (l) O. Illa, M. Arshad, A. Ros, E. M. McGarrigle and V. K. Aggarwal, J. Am. Chem. Soc., 2010, 132, 1828-1830; (m) B.-H. Zhu, J.-C. Zheng, C.-B. Yu, X.-L. Sun, Y.-G. Zhou, Q. Shen and Y. Tang, Org. Lett., 2010, 12, 504-507; (n) V. K. Aggarwal, J. P. H. Charmant, D. Fuentes, J. N. Harvey, G. Hynd, D. Ohara, W. Picoul, R. Robiette, C. Smith, J.-L. Vasse and C. L. Winn, J. Am. Chem. Soc., 2006, 128, 2105-2114; (o) D. Janardanan and R. B. Sunoj, J. Org. Chem., 2008, 73, 8163-8174; (p) V. K. Aggarwal and J.-L. Vasse, Org. Lett., 2003, 5, 39873990; ( $q$ ) V. K. Aggarwal, J. G. Ford, S. Fonquerna, H. Adams, R. V. H. Jones and R. Fieldhouse, J. Am. Chem. Soc., 1998, 120, 8328-8339.

8 (a) J.-C. Zheng, C.-Y. Zhu, X.-L. Sun, Y. Tang and L.-X. Dai, J. Org. Chem., 2008, 73, 6909-6912; (b) L.-Q. Lu, J.-J. Zhang, F. Li, Y. Cheng, J. An, J.-R. Chen and W.-J. Xiao, Angew. Chem., Int. Ed., 2010, 49, 4495-4498; (c) Q. Wang, X. Qi,
L.-Q. Lu, T.-R. Li, Z.-G. Yuan, K. Zhang, B.-J. Li, Y. Lan and W.-J. Xiao, Angew. Chem., Int. Ed., 2016, 55, 2840-2844; (d) Q.-Q. Yang, Q. Wang, J. An, J.-R. Chen, L.-Q. Lu and W.-J. Xiao, Chem.-Eur. J., 2013, 19, 8401-8404; (e) L.-Q. Lu, Y.-J. Cao, X.-P. Liu, J. An, C.-J. Yao, Z.-H. Ming and W.-J. Xiao, J. Am. Chem. Soc., 2008, 130, 6946-6948; (f) J.-R. Chen, W.-R. Dong, M. Candy, F.-F. Pan, M. Jörres and C. Bolm, J. Am. Chem. Soc., 2012, 134, 6924-6927; (g) P. Xie, L. Wang, L. Yang, E. Li, J. Ma, Y. Huang and R. Chen, J. Org. Chem., 2011, 76, 7699-7705.

9 (a) X. Tang, H.-P. Zhu, J. Zhou, Y. Chen, X.-L. Pan, L. Guo, J.-L. Li, C. Peng and W. Huang, Org. Biomol. Chem., 2018, 16, 8169-8174; (b) S. Roy and V. Piradhi, ChemistrySelect, 2017, 2, 6159-6162; (c) Y. Li, Q.-Z. Li, L. Huang, H. Liang, K.-C. Yang, H.-J. Leng, Y. Liu, X.-D. Shen, X.-J. Guo and J.-L. Li, Molecules, 2017, 22, 328; (d) X.-S. Meng, S. Jiang, X.-Y. Xu, Q.-X. Wu, Y.-C. Gu and D.-Q. Shi, Eur. J. Org. Chem., 2016, 2016, 4778-4781; (e) A. M. Bernard, A. Frongia, P. P. Piras, F. Secci and M. Spiga, Org. Lett., 2005, 7, 4565-4568; $(f)$ E. J. Corey and M. Chaykovsky, J. Am. Chem. Soc., 1962, 84, 867-868.

10 (a) A. A. Volkers, X. S. Mao, A. J. H. Klunder and B. Zwanenburg, Tetrahedron, 2009, 11, 2364-2367; (b) S. E. Sheikh, N. Kausch, J. Lex, J.-M. Neudorfl and H.-G. Schmalz, Synlett, 2006, 10, 1527-1530; (c) J. T. Blanchfield, S. Chow, P. V. Bernhardt, C. H. L. Kennard and W. Kitching, Aust. J. Chem., 2004, 57, 673-676.

11 (a) J. Chen, P. Jia and Y. Huang, Org. Lett., 2018, 20, 67156718; (b) R. Oost, J. D. Neuhaus, A. Misale, R. Meyrelles, L. F. Veiros and N. Maulide, Chem. Sci., 2018, 9, 70917995; (c) C. Li, K. Jiang, Q. Ouyang, T.-Y. Liu and Y.-C. Chen, Org. Lett., 2016, 18, 2738-2741.

12 (a) X. Xie, W. Huang, C. Peng and B. Han, Adv. Synth. Catal., 2018, 360, 194-228; (b) Q. Zhao, C. Peng, H. Huang, S.-J. Liu, Y.-J. Zhong, W. Huang, G. He and B. Han, Chem. Commun., 2018, 54, 8359-8362; (c) M.-C. Yang, C. Peng, H. Huang, L. Yang, X.-H. He, W. Huang, H.-L. Cui, G. He and B. Han, Org. Lett., 2017, 19, 6752-6755; (d) X. Li, W. Huang, Y.-Q. Liu, J.-W. Kang, D. Xia, G. He, C. Peng and B. Han, J. Org. Chem., 2017, 82, 397-406; (e) B. Han, W. Huang, W. Ren, G. He, J.-H. Wang and C. Peng, Adv. Synth. Catal., 2015, 357, 561-568; (f) R. Zhou, Q.-J. Wu, M.-R. Guo, W. Huang, X.-H. He, L. Yang, F. Peng, G. He and B. Han, Chem. Commun., 2015, 51, 13113-13116; (g) X. Li, L. Yang, C. Peng, X. Xie, H.-J. Leng, B. Wang, Z.-W. Tang, G. He, L. Ouyang, W. Huang and B. Han, Chem. Commun., 2013, 49, 8692-8694; $(h)$ X. Xie, C. Peng, G. He, H.-J. Leng, B. Wang, W. Huang and B. Han, Chem. Commun., 2012, 48, 10487-10489; (i) B. Wang, H.-J. Leng, X.-Y. Yang, B. Han, C.-L. Rao, L. Liu, C. Peng and W. Huang, RSC Adv., 2015, 5, 88272-88276.

13 CCDC 1895883 (for 6r) contains the supplementary crystallographic data for this paper. $\dagger$

14 CCDC 1895884 (for 10a) contains the supplementary crystallographic data for this paper. $\dagger$ 